\title{
SINGLE TROJAN INJECTION MODEL GENERATION AND DETECTION
}

\author{
by \\ HARINI BHAMIDIPATI
}

\author{
Submitted in partial fulfillment of the requirements \\ For the degree of Master of Science
}

Thesis Advisor: Dr. Daniel G. Saab

Department of Electrical Engineering and Computer Science

CASE WESTERN RESERVE UNIVERSITY

January 2010 


\section{Table of Contents}

1 Introduction $\quad 1$

1.1 Advantages of Outsourcing . . . . . . . . . . . . . . . 2

1.2 Disadvantages of Outsourcing . . . . . . . . . . . . . . . 4

2 Background $\quad 6$

2.1 Classification of Trojans . . . . . . . . . . . . . . . . 6

2.2 Challenges in the detection of Trojans . . . . . . . . . . . . . . . 10

2.3 Related Prior Work . . . . . . . . . . . . . . . . . . 12

2.4 Contribution of this thesis: . . . . . . . . . . . . . . . . 15

3 Modeling of Trojans $\quad 16$

3.1 Distance Between two Nodes: . . . . . . . . . . . . . . . . . . . . . 16

3.2 Trojan Circuit Modeling: . . . . . . . . . . . . . . . . . . . 17

4 Detection of Trojans $\quad 22$

4.1 ATPG and its operation: . . . . . . . . . . . . . . . 22

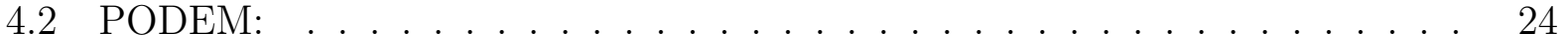

4.3 ATPG for Sequential Circuits: . . . . . . . . . . . . . . 26 
4.4 Detection Scheme for Trojans: . . . . . . . . . . . . . . . 27

4.4.1 Modified PODEM Algorithm ................ 28

5 Results $\quad 37$

5.1 Analysis of generated patterns . . . . . . . . . . . . . . 38

5.2 Fault Simulation . . . . . . . . . . . . . . . . . . 38

5.2.1 Trojan Insertion Model . . . . . . . . . . . . . . . . . 42

5.2.2 Results of Fault Simulation . . . . . . . . . . . . . . 45

$\begin{array}{llr}6 & \text { Conclusion } & 47\end{array}$ 


\section{List of Tables}

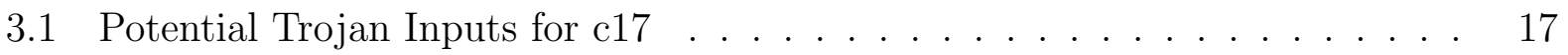

5.1 Iscas85: Modeled Trojan . . . . . . . . . . . . . . . . . . . 39

5.2 Iscas89: Modeled Trojan $\mathrm{k}=3$. . . . . . . . . . . . . . . . . . . 40

5.3 Iscas85: Modeled Trojan $\mathrm{k}=2 \ldots \ldots$. . . . . . . . . . . . . 41

5.4 Iscas85: Trojan Detection . . . . . . . . . . . . . . . 46

5.5 Iscas89: Trojan Detection . . . . . . . . . . . . . . 46 


\section{List of Figures}

1.1 Old Supply Chain Structure . . . . . . . . . . . . . . . . . 2

1.2 New Supply Chain Structure . . . . . . . . . . . . . . . . . 3

2.1 Classification of Trojans . . . . . . . . . . . . . . . . 7

3.1 Injection of a 4 -input 1 -output Trojan into c17 . . . . . . . . . . . . 18

3.2 Injection of a 4 -input 1 -output Trojan into c17 . . . . . . . . . . . . 19

4.1 Detection of stuck at faults . . . . . . . . . . . . . . . . . 23

4.2 Decision Tree . . . . . . . . . . . . . . . . . . 25

4.3 High Level Representation of PODEM Algorithm . . . . . . . . . . . . . . . 31

4.4 Iterative Logic Array . . . . . . . . . . . . . . . . . . . . . . . . . . . . 32

4.5 Circuit with Trojan Injected . . . . . . . . . . . . . . . . . 32

4.6 Test pattern generation for Trojan . . . . . . . . . . . . . . . . . 33

4.7 Test pattern generation for Trojan . . . . . . . . . . . . . . . 33

4.8 Test pattern generation for Trojan . . . . . . . . . . . . . . . . . 34

4.9 Test pattern generation for Trojan . . . . . . . . . . . . . . . . 34

4.10 Test pattern generation for Trojan . . . . . . . . . . . . . . . 35

4.11 Modified PODEM Algorithm for the detection of Trojans . . . . . . . . . 36 


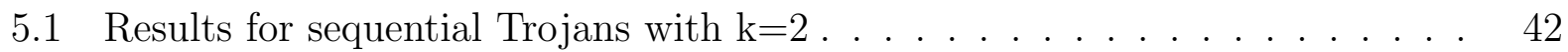

5.2 Results for sequential Trojans with $\mathrm{k}=3 \ldots \ldots$. . . . . . . . . . . . 42

5.3 Observability and controllability values . . . . . . . . . . . . . 44 


\section{Acknowledgments}

I would like to thank and express sincere gratitude to my adviser Dr. Daniel G. Saab for his guidance and support in completing this work. His wide knowledge and expertise has been essential to complete my thesis. His guidance, encouragement and supervision are invaluable and have helped me throughout my master's program. It has been a pleasure working with him.

I am indebted and thankful to Prof. Christos Papachristou and Prof. Francis Merat for their support as members of my thesis defense committee

Many thanks to my family and friends for all the support and encouragement they have provided.Lastly I would like to thank the staff at EECS department and International Student Services for making my life at CWRU comfortable. 


\section{Single Trojan Injection Model Generation and Detection}

Abstract

by

\section{HARINI BHAMIDIPATI}

This thesis proposes a technique for non-destructive and functional testing of ICs to detect modifications made to the design. The outsourcing of the fabrication process by semiconductor manufacturers has made IC design vulnerable to third party modifications and placed a risk on the reliability and security of hardware systems. These hardware modifications/ Trojans are carefully injected so that they cannot be detected using regular functional testing techniques. Once injected, a Trojan can perform any functionality from resetting the circuit to transmitting signals to propagating incorrect signals to the circuit output.

In this work we present a technique for injection, modeling and detection of Trojans. Here the inputs of a Trojan are identified using proximity estimation techniques and the Trojan is modeled as a black box with the identified set of IOs. Then the test patterns for the detection of Trojans are generated using a modified form of ATPG. In our experiments we generated the test patterns for combinational and sequential Trojans in various benchmark circuits. Then we injected Trojans in some of the circuits and performed fault simulation to test the efficiency of the generated patterns. 


\section{Chapter 1}

\section{Introduction}

Globalization resulted in diminution or elimination of state-enforced restrictions on ex-

changes across borders and the formation of increasingly integrated and complex global system of production and exchange. As such, the mobility of factors of production (capital, labor, goods and services, and technology) increased. The opening of economies by many developing countries has further accelerated globalization. Thus there was a shift to open market economy, encouraging private and international investments and allowing economic agents to operate without undue regulations.

The semiconductor industry was not insulated from the changes in the economies and markets, and had to follow the trend. The increasing product development costs along with advancing technology forced many companies to follow the trend. To survive the competition and to take advantage of the various incentives and low labor costs offered by other countries there was a huge restructuring of the organizations.Companies started outsourcing many of their activities. They transformed the structure of their organizations 


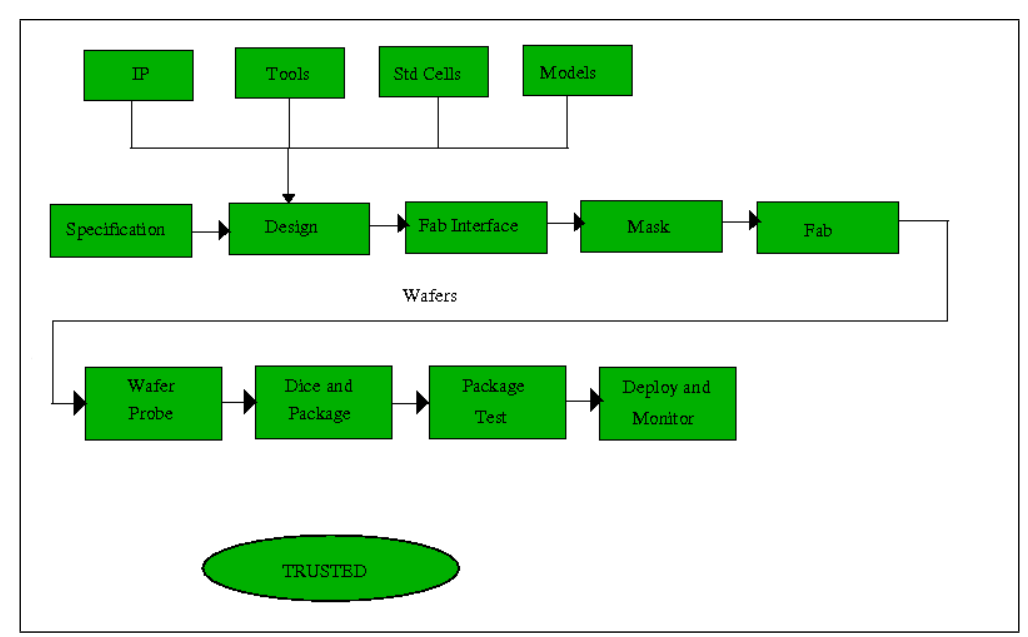

Figure 1.1: Old Supply Chain Structure

from a vertical integrated company structure to a horizontal consolidation. Figures fig1.1 and fig 1.2 [originally presented in [4]] show the difference in the organization structures. The fabrication of the ICs, being the one of the most expensive part of the IC design was outsourced.

The restructuring of organization, though offered many advantages had its own share of disadvantages.

\subsection{Advantages of Outsourcing}

- Compression of the development cycle for new products: The third party firms that offer specific services, can improve their methodologies over time to shorten the product development cycle and to produce a cost effective, efficient engineering outcome. Thus by outsourcing the companies can take advantage of these improved methodologies. 


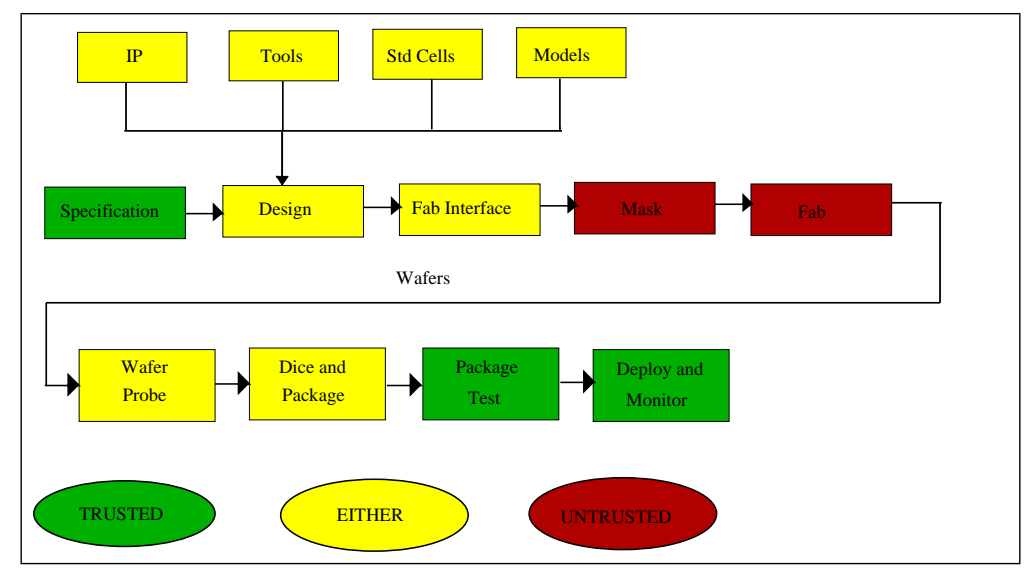

Figure 1.2: New Supply Chain Structure

- Flexibility: The company can concentrate on its core competency product. The development cycle of the third party firm occurs in parallel with the product development in the outsourcing firm. Hence the outsourcing firm can respond to the market/competitive changes as needed.

- Operational efficiency: Outsourcing can relieve a company of investing in fixed assets, additional engineering resources and additional labor that is needed to take the core product to the market. Conversely, it can allow a company to invest in additional resources to develop their core competencies.

- Utilization of external engineering resources: The development of a product can require engineering talents that do not exist within a company's resources. Rather than invest in additional resources, which will need to be trained and acclimated into the existing engineering team, it is an advantage to outsource these engineering projects.

- Beat Competition: For a semiconductor company to be successful it needs to provide 
high-quality services at cheap prices. Outsourcing would help the company maintain lower rates with better service solutions, thereby giving them a better market position or even a competitive advantage. This restructuring has resulted in the global dispersion of the manufacturing operations.

\subsection{Disadvantages of Outsourcing}

Outsourcing has many commercial advantages and over the period it is emerged as a lucrative option for semiconductor manufacturing firms to ensure their competitive edge in the market. However there are some disadvantages in this new supply chain mechanism. As the third party manufacturing units are usually located overseas it is not possible to monitor the fabrication processes and practices. This results in most of the disadvantages of outsourcing.

- Dependency of the time to market on the third party manufacturer:As part of the circuit design/ manufacturing activity carried out by the third party service provider, the time to market a product depends on the service provider. Hence the outsourcing company has to rely on the third party manufacturer to meet the deadlines.

- Intellectual Property (IP) Rights violation: To complete the fabrication process the design details of the IC are revealed to the manufacturer. Once provided with the design details the third party manufacturer can reverse engineer the design and fabricate similar products to sell at a lower price. This affects the net consumption of the product once released in the market.

- Risk of security and trust: Also with the availability of the design details the manufacturer can modify the design for malicious purposes. This modification can 
be brought about by inserting/ removing design circuitry. This additional circuitry is called the Trojan circuitry.

Here in this thesis we are concerned with the security and reliability issue of ICs caused by the insertion of Trojans. Trojans are intelligently injected in the circuit to activate in rare events and cause catastrophic effects [24]. A Trojan can have any functionality from resetting the circuit to transmitting signals that help in tracking the circuit remotely to propagating incorrect signals to the circuit output. Therefore Trojan designs are a cause of great concern for anyone using ICs [13],[18].Many methods have been proposed to detect Trojans and improve the reliability and security of ICs. The details of these techniques are mentioned in chapter 2. This thesis presents a new technique to detect Trojans using a modified ATPG. The details of this technique are mentioned in chapters [3] and [4]. Chapter[3] explains the modeling of Trojans and chapter[4] enunciates the details of the Trojan detection technique. Test patterns for various benchmark circuits were generated using this technique and fault simulation is performed. The results of the test pattern generation and fault simulation are presented in chapter[5]. 


\section{Chapter 2}

\section{Background}

Trojans are small circuits that occupy very small percentage of the chip area. They are mostly inactive during the normal operation of the chip. They are placed on nodes that are hard to detect ie., those nodes that are not easily observable or controllable. This makes it difficult to detect them using regular functional testing strategies. Once activated they disrupt the normal operation of the chip. Trojans can vary from those that are inserted by the parametric variations to those that are implemented by inserting additional logic. Techniques have been developed to detect different kinds of Trojans. This chapter gives a description of various types of Trojans based on their general characteristics. It also defines the various terms used in this thesis.

\subsection{Classification of Trojans}

Trojans can be broadly categorized as combinational Trojans and sequential Trojans. Combinational Trojans are the Trojans that are activated in a specific condition or for a combi- 


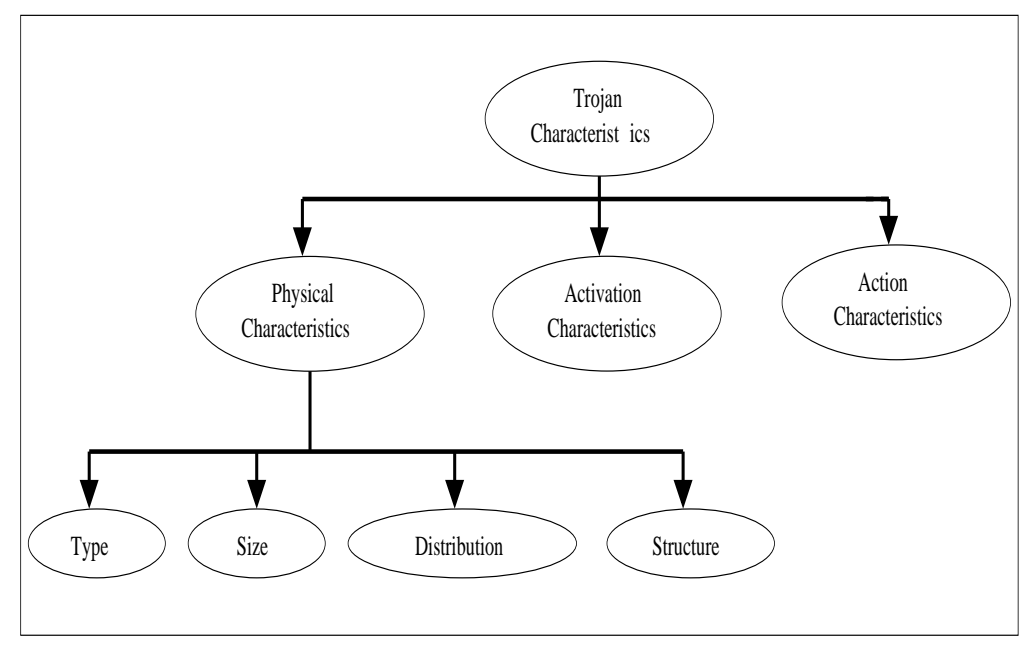

Figure 2.1: Classification of Trojans

nation of values on a set of nodes in the circuit. Sequential Trojans are like a FSM and are activated for a specific sequence of input signals.

Apart from the broad classification mentioned above, Trojans can be further classified [The classification was first proposed in [6]] based on their physical characteristics, activation characteristics and action characteristics. This classification is represented in figure 2.1Physical characteristics can be further classified based on the type, size, distribution and structure.

\section{- Physical characteristics of Trojan:}

Below is a description of the various physical characteristics of the Trojan.

- Type: There are two types of Trojans, functional and parametric Trojans. Functional Trojans are those that modify the design by the addition or deletion of logic elements like the transistors and logic gates. Parametric Trojans are formed 
by the modification of the physical geometry of the wire or the logical elements (transistors). They can be formed by thinning a wire, weakening of a transistor etc.

- Size: Size of a Trojan is an important factor in the detection of Trojans. Smaller Trojans are difficult to activate and produce small variations in the side channel signals. Hence, smaller Trojans are difficult to detect using side channel analysis techniques.

- Distribution: The distribution of a Trojan is a measure of the degree of dispersion of the components of a Trojan. The distribution is mainly determined by the availability of spaces in the design. If the design has very small spaces available, the adversary will be forced to distribute the Trojan circuitry in a larger area.

- Structure: The Trojans that result in a change in the circuit structure/layout are easily detectable. They usually change the placement of other components of the design and hence produce a significant variation in the IC fingerprint. With the variation in the IC fingerprint, they can be detected by the side channel analysis techniques. To prevent detection, the large Trojans can be distributed in the design to reduce the variation of the side channel signals locally. However, distribution of the components varies other physical aspects of the design (For example, the amount of wiring in the design) which in turn results in the variation of the IC fingerprint. Hence, small tightly packed Trojans which cannot be detected easily are more attractive.

- Activation Characteristics of Trojans: Trojans are activated intelligently by the 
adversary in rare conditions to implement a disruptive function. Trojan can be activated using external signals or by internal signals.

- Externally activated Trojans: Trojans can be activated by mounting a receiver or an antenna in the chip and controlling it using external signals. The Trojan can thus be activated at a time desired by the adversary.

- Internally activated Trojans: They are of two types, the always-on Trojans and conditionally-on Trojans.

* Always-on Trojans: They are always active and can influence the operation of the circuit at any time desired by the adversary. All the parametric Trojans belong to this criteria. As the Trojan is always on, they are hidden by the adversary by placing them on nodes or paths that are hard to detect. As long as these paths are not exercised, the circuit behaves like a Trojan free circuit. Hence the Trojan is invisible or undetectable by regular functional testing patterns.

* Conditionally-on Trojans: These are activated on the occurrence of specific conditions. For example, at a specific temperature or on the occurrence of a specific set of input patterns. They have additional logic components and are not just the parametric variations. This additional logic can be detected by functional testing.

\section{- Trojan Action Characteristics:}

Depending on the disruptive action performed by the Trojans they can be classified as modify specification, modify function and transmit information Trojans. 
- Modify specification: These Trojans cause a variation in the specification of the circuit. For example they can cause a variation in the delay of the circuit etc. They do not modify the functionality of the chip but they cause a system failure by varying the specifications.

- Modify function: These Trojans modify the functionality of the chip by inserting additional logic or by modifying the existing logic. As they change the functionality of the chip they can implement additional functionality.

- Transmit Information: These Trojans transmit information from the design to the adversary. They do not modify the functionality of the chip but rather use the side channel signals to transmit information to the adversary.

\subsection{Challenges in the detection of Trojans}

As discussed above Trojans are intelligent circuits that are active under rare specific conditions. It is very difficult to detect them using regular functional testing patterns. Consider a n input Trojan. Assume a probability of $P_{i}$ in justifying a $0 / 1$ on the $i^{t h}$ input of the Trojan circuit. When the Trojan circuit is designed care is taken to keep the value of this probability very low to minimize the probability of Trojan detection. To detect a Trojan using functional testing the Trojan should be activated and then the output of the Trojan should be propagated to the primary outputs of the circuit. Hence the probability of detecting a Trojan is 


$$
\begin{aligned}
& \mathrm{P}=\mathrm{P}(\text { activation }) \cdot \mathrm{P}(\text { propagation }) \text { where } \\
& \qquad P(\text { activation })=\prod_{i=1}^{n}\left(P_{i}\right)
\end{aligned}
$$

Let the number of inputs to the Trojan be 10. Assuming a low probability value of $10^{-3}$ for justifying the Trojan inputs, the probability of activating a Trojan is $10^{-30}$. If the probability of propagating the Trojan's effect is also considered the probability of detecting Trojans is further worsened.

In the side channel analysis methods the major challenge would be to produce a variation in the side channel behavior that is greater than the variation produced by the process variations. This usually limits the type of Trojans detected by these methods to large Trojans that consume more power and which can be easily activated. However, this problem can be overcomed to certain extent by generating patterns that maximize switching current in a small region and minimize the switching current in the remaining region of the circuit. Paper [1] presents a method to generate such patterns and maximize the chances of detection.

From the above discussion we can see that both the methods have different advantages and any one particular method cannot be used to detect all types of Trojans. Different strategies have to be adopted to detect different types of Trojans. As the type and size of Trojans is not known before testing, it is essential to use both the methods to increase the probability of detection. It is considered that the adversary will insert Trojans randomly in a large batch of chips. Also, when selecting methods for detection of Trojans it is important to consider the time taken for testing each chip using that method. 


\subsection{Related Prior Work}

Recently methods have been proposed for the detection of Trojans. Most of the proposed techniques are based on the analysis of the side channel signals. Some of the techniques involve using destructive reverse engineering techniques to collect the side channel signals. In large ICs the amount of area that a Trojan occupies is less than $0.01 \%$ of the total chip

area. Hence, the variation that they produce in the side channel signals might be too small to be detected using side channel analysis techniques. However, these techniques can be used to detect large Trojans that cause significant changes in the side channel behavior. Bellow is a brief description of the various techniques proposed till date. This chapter also provides the motivation and overview of the technique proposed in this thesis.

- In [7] a current integration method has been proposed for Trojan detection and isolation. In this method first a golden chip is identified using exhaustive test from a number of randomly selected chips. It is assumed that Trojans are introduced randomly in a selected number of chips and not in all the chips. Then the worst case charge is obtained in response to the pattern set. The worst case charge is based on worst case process variations in one of the genuine ICs. Next, the pattern set is applied to each of the manufactured chip and the current is measured locally via power ports. Then the charge variations with time are calculated for all the current waveforms obtained after applying the patterns. When applying patterns the charge increases and is compared continuously against the worst case charge calculated for golden chips. Once the difference between the two curves $\delta Q$ is greater than $Q_{t h r}$ (a charge threshold value which is a resolution measurement defined by the instrumentation) the Trojan 
is considered to be detected. However in the nanoscale technologies the size of the Trojan circuit is much smaller and its effect on the side channel signal might be too small to be detectable.

- In [4] a side channel based fingerprinting methodology for detecting Trojan ICs is proposed. In this method first ICs of the same family are selected (ie., the ICs using the same mask and manufactured in the same fab). Then all possible I/O tests are applied multiple times and one or more side-channel signals (power, EM, Thermal Emissions etc.) are calculated for the ICs during these tests. All these side channel signals are used to build a side channel fingerprint for the IC family. Also the selected ICs are destructively validated to meet the original specifications. Once the finger prints are collected all the ICs manufactured can be subjected to the same test vectors and the resulting side channel signals can be compared to be consistent with the fingerprints. The fingerprints are generated based on the process variations during the manufacture of the ICs. Here the maximum process variation is considered to be $7.5 \%$, however in the nanoscale technologies the process variations can be more than that, thus making it difficult to detect the Trojans. Also, this method requires an additional IC fingerprint generation and validation step to be carried out by a trust worthy IC testing facility.

- In [2] a method is proposed to use the power profile of the circuit to detect Trojans. First the circuit is divided into regions based on the structural connectivity. As Trojans usually form only a small fraction of the overall circuit, the relative increase in the circuit activity due to the presence of a Trojan is also small. It might be difficult to observe the difference in the power profile for some circuits. Hence in this method the test vectors are generated based on the state elements such that they maximize the 
activity in the Trojan portion of the circuit and minimize in the remaining portion of the circuit. Then the relative difference in the power profiles of a genuine and Trojan circuit are used to detect Trojans. However, Trojans are intelligent circuits which are activated on certain conditions. Increasing the hamming distance may not necessarily activate the Trojan and hence may not cause any difference in the power profiles of a genuine and Trojan circuit.

- In [1] a method is proposed to identify Trojans based on the power profiles of certain regions in a circuit. The regions are obtained based on the radius from a gate. In this method only those regions are considered that contain a threshold number of flip flops. Once the regions are identified the circuit is simulated with vectors that maximize the switching activity within the region of interest while reducing the activity in the rest of the circuit. Then the difference in the power profiles of genuine and Trojan ICs in the selected region is used for identification of Trojans.

- In [3] a new logic testing scheme for detecting Trojans has been proposed. It is based on the frequency of the rare values which trigger the Trojan. Here first a Trojan target analysis procedure is conducted to identify the inputs and outputs of the Trojan, trigger values and frequencies of each of the Trojan circuits and the trigger vectors associated with the trigger values for the Trojans. In the Trojan target analysis the Trojan inputs are identified as all combinations of edges that attain one or more bit values with frequency $\leq f_{t h}$. The outputs of the Trojan are all edges that have low probability of propagating the fault to the circuit output. Then the Trojan detection procedure is carried out using an ATPG tool to check if the input trigger vector can be propagated to the circuit output. 
- In [5] the authors provide a method of generating physical unclonable functions from the physical characteristics of ICs. PUFs use the random variations during the IC fabrication process. They are volatile secrets that exits only in a digital form when the IC is on and are not stored as non volatile secrets in the digital memory. The inputs fed to the PUFs are termed as challenges and the outputs that they produce are termed as responses. The unique way of mapping the challenges and responses makes the PUFs unclonable. Also, PUFs are efficient in preventing external attacks but Trojan attacks are on-chip intrusions and hence require different methods to detect them.

\subsection{Contribution of this thesis:}

Here in this thesis we propose a method for modeling Trojans and generating test patterns for the detection of Trojans. The modeling of Trojans is based on proximity estimation measures that identify potential ways a Trojan can be inserted into a specific design location. Once the Trojan is modeled a modified Automatic Test Pattern Generator is used for the detection of Trojans. The advantages of this modeling and test generation procedure are 1.) it does not require any modifications to the existing design flow, 2.) It uses existing ATPG tools, 3.) It can be applied to all ICs uniformly, 4.) it can detect small as well as large Trojans. 


\section{Chapter 3}

\section{Modeling of Trojans}

In modeling Trojans the inputs and outputs are identified using proximity estimation measures. This chapter first explains the procedure for calculating the distance between two nodes, then the details of modeling Trojans are presented with examples.

\subsection{Distance Between two Nodes:}

The distance between two nodes is calculated based on the topology of the circuit. First, all the nodes in the immediate fanin/fanout of the selected node are assigned a distance of 1. Next, all the nodes in the immediate fanin/fanout of the nodes with distance 1 are assigned a distance 2.This procedure can be repeated to find the nodes with distance greater than 2 from the selected node. For example, consider node 10 of the $\mathrm{C} 17$ circuit shown in Fig3.1. The nodes $\{1,3,22\}$ are in the immediate fanin/fanout of the node 10 and are assigned a distance of 1 . Now, all nodes in the immediate fanin/fanout of the nodes $\{1,3,22\}$ are listed and a distance of 2 is assigned to them. Hence, the nodes $\{11,16\}$ are at a distance 
Table 3.1: Potential Trojan Inputs for c17

\begin{tabular}{|c|c|c|}
\hline Trojan node & $\mathrm{d}=1$ & $\mathrm{~d}=2$ \\
\hline \hline 1 & $\left\{10^{*}\right\}$ & $\left\{3,22^{*}\right\}$ \\
\hline 2 & $\left\{16^{*}\right\}$ & $\left\{11,22^{*}, 23^{*}\right\}$ \\
\hline 3 & $\left\{10^{*}, 11^{*}\right\}$ & $\left\{1,6,19^{*}, 16^{*}, 22^{*}\right\}$ \\
\hline 6 & $\left\{11^{*}\right\}$ & $\left\{3,16^{*}, 19^{*}\right\}$ \\
\hline 7 & $\left\{19^{*}\right\}$ & $\left\{11,23^{*}\right\}$ \\
\hline 10 & $\left\{1,3,22^{*}\right\}$ & $\{11,16\}$ \\
\hline
\end{tabular}

of 2 . This procedure can be repeated to find all the nodes with a distance greater than 2 . The nodes that are at a distance of 1 and those that are at a distance 2 are listed for few nodes of the circuit $\mathrm{C} 17$ in Table 3.1.

\subsection{Trojan Circuit Modeling:}

Trojan modeling scheme presented here does not provide the details of the structure of the Trojan, but identifies the possible set of inputs and outputs for a Trojan. Then the Trojan is treated as a black box with unknown functionality, unknown number of inputs and outputs, but with an identified set of all possible inputs and outputs. As we only need the set of input patterns that produce an error at the output, the information about the structure of the Trojan is not essential for the detection process. Large Trojans cause a detectable variation in the side channel behavior and can be detected by the techniques proposed in [7], [4], [2], [14], [21], [22], [23], [24], [25], [26], [27], [28], [29] and [1]. Here we try to detect small Trojans. In our experiments we assumed the number of inputs to the Trojan to be $\leq 5$.

To model small Trojans we restrict the number of inputs and outputs using the following heuristics. 
- Topology Driven: The Trojan inputs are selected from the circuit nets based on the closeness/distance of the Trojan inputs from the Trojan outputs. Only those nets whose distance is less than or equal to a maximum distance of $d_{\max }$ are selected.

- Controllability/ Observability Driven: Only those nets that are hard to control are selected as the Trojan inputs. If the topology driven heuristic selects more than MAXI nets, then the controllability/ Observability driven heuristic is used to further limit the number of inputs.

To model Trojan on a given node $c_{j}$, first all the nodes that are at a distance $\mathrm{n}$ from $c_{j}$ are identified. Out of the identified nodes only those nodes that do not cause a feedback are considered as inputs to the Trojan. Then a black boxed Trojan is inserted with the identified set of inputs and outputs on node $c_{j}$. This can be clearly understood by looking at the following example.

\section{Example1: To model Trojan on node 10 of circuit C17}

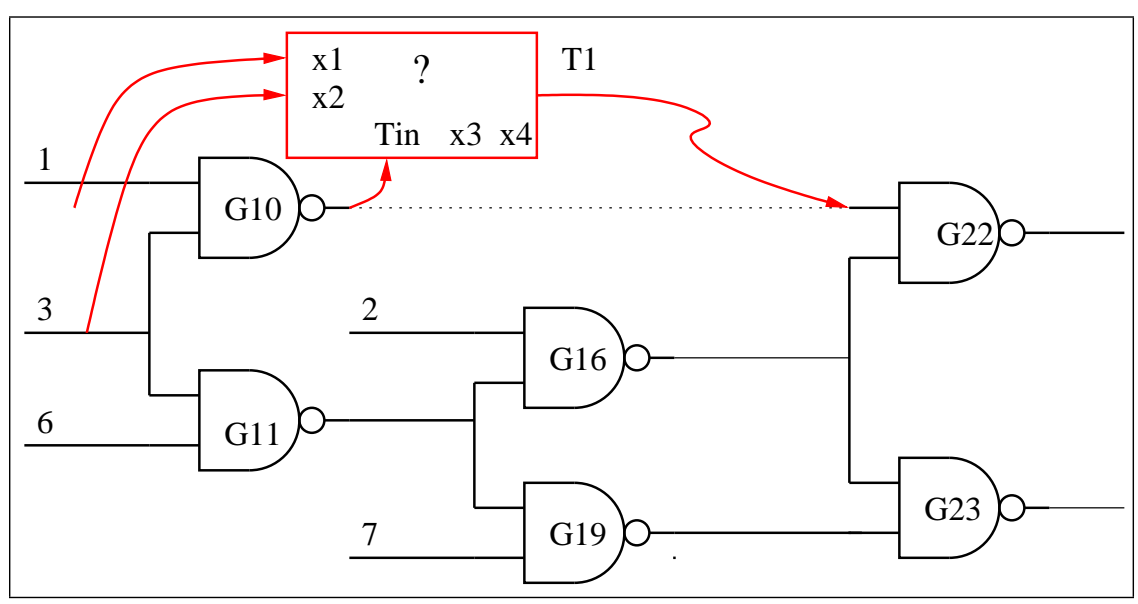

Figure 3.1: Injection of a 4-input 1-output Trojan into c17 
- Case 1: Consider the inputs to the Trojan to be at a maximum distance of 1 from node 10. The nodes that are at distance 1 from node 10 are $\{1,3,22\}$. Out of these nodes, node 22 cannot be considered as an input to the Trojan as it results in a dependency/ feedback. The possible set of inputs to the Trojan is $\{1,3\}$. Then the net connecting G10 to G22 is disconnected (shown as dashed line in Fig 3.2), and a single black boxed Trojan is injected at node 10. The resulting circuit is shown in Fig 3.1.

- Case 2: Consider the inputs to the Trojan to be at a maximum distance of 2 from node 10. Only nodes $1,3,11,16$ satisfy the condition. Hence we model the Trojan as shown in Fig 3.2.

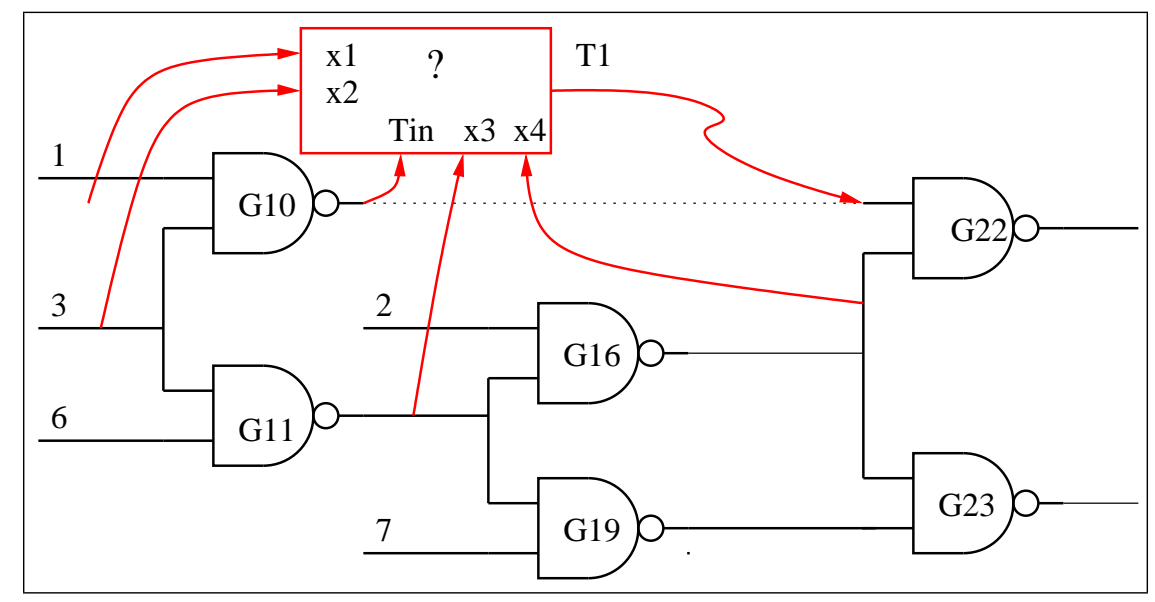

Figure 3.2: Injection of a 4-input 1-output Trojan into c17

Note: This method of modeling Trojans always has one output only. However, it helps in detecting Trojans with multiple outputs. For example: consider the Trojan in case 2 to have two outputs one at node 10 and another at node 16. This Trojan can be detected by the patterns generated for Trojan at node 10 and by those for node 16 . 
This procedure can be applied for cases when the distance of the Trojan inputs from the node on which the Trojan is inserted is greater than 2. However, as the distance increases, the amount of wiring needed to inject the Trojan increases, leading to a significant variation in the side channel signal behavior. Such Trojans can be detected using the techniques proposed in [7], [4], [2] and [1].

The Trojan circuit receives its inputs from the main circuit and feeds its output to the main circuit. Hence, the total number of $\mathrm{I} / \mathrm{O}$ nets is bounded by the number of nets in the main circuit. For a circuit with q nets the total number of inputs and outputs for the Trojan is at most $\left(\begin{array}{c}q \\ |T P I|\end{array}\right) *\left(\begin{array}{c}q \\ |T P O|\end{array}\right)$. In our experiments we assumed that for sequential circuits the Trojans cannot be inserted on the inputs/outputs to a flip-flop. The following points should be noted about this method of modeling Trojans

- The Trojans can be modeled if

- The number inputs and outputs is bounded

- inputs are connected to a set of nets in the main circuit

- outputs assume the function of nets in the main circuit

- Only the set of I/O nets of the Trojans are identified, the order of mapping of these nets to the primary inputs/outputs of the Trojan circuit is not considered. As the functionality of the Trojan is unknown, considering the permutation of the input nets only results in another unknown functionality. This new functionality can be assumed to be the functionality of the Trojan.

- When modeling Trojans the set of all possible inputs are selected, hence if the actual number of inputs is less than the set of inputs considered for the Trojan, the extra 
inputs behave as don't care inputs. Similarly, if the actual number of outputs considered for the Trojan is less than the number of modeled outputs, these extra outputs behave identical to the nets to which they are mapped. Also, the Trojan clock can be connected to the main circuit clock or to any other net of the main circuit. 


\section{Chapter 4}

\section{Detection of Trojans}

As seen from the Trojan model presented in chapter 3, a Trojan has $n$ inputs and one output. A Trojan is said to be detected if the inputs of the Trojan can be justified with the activation pattern and the if Trojan output can be propagated to the Primary Output of the circuit.

The justification and activation are similar to the detection of a saf using an ATPG. However the ATPG needs to be modified to accommodate the differences in a saf and a Trojan. This chapter first discusses about the operation of a ATPG and the PODEM algorithm [11] and then about modified ATPG and the detection of Trojans.

\subsection{ATPG and its operation:}

Automatic test pattern generator generates stimuli for a stuck at fault. The first step in the test generation process is the justification. Once the error is justified it is propagated to the output. If the decision leads to inconsistent values (during the implication or in the future justification/propagation phases), we trace back to our last decision point and make a new 
decision, this process is repeated until the inconsistency is resolved or the search space is exhausted. for example, consider the circuit shown in figure $4.1^{1}$ with stuck at fault at $\mathrm{h}$.

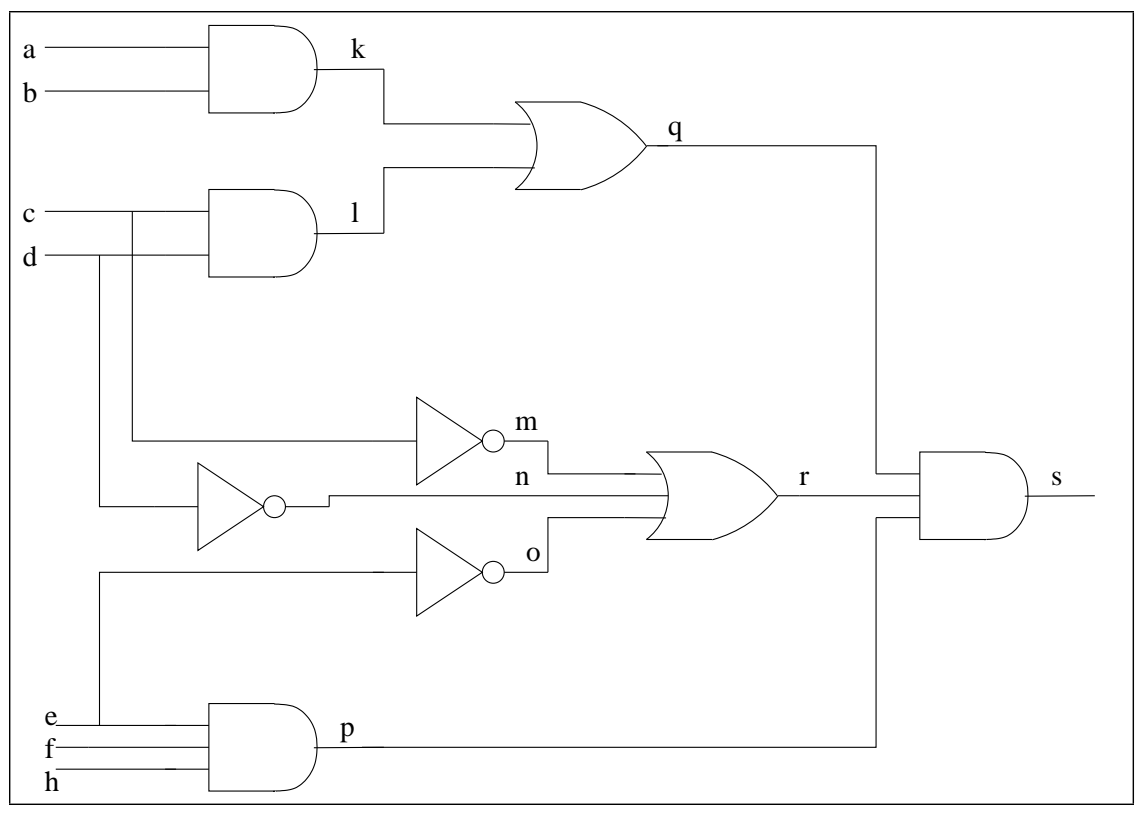

Figure 4.1: Detection of stuck at faults

Objective: need to generate the test patterns for the stuck at fault $h=1$.

- To generate the test patterns for the saf, we need to justify a 0 at $\mathrm{h}$. Then we assign the remaining inputs of the gate to non-controlling values. Hence $e=1$ and $f=1$.

- To propagate the value to the output it is required that $q=1$ and $r=1$, we now need to justify these values.

- $q=1$ can be justified by assigning either $\mathrm{l}$ or $\mathrm{k}$ to 1 .

\footnotetext{
${ }^{1}$ This example is taken from Digital Systems Testing and Testable Design by Miron Abramovici, Melvin A. Breuer and Arthur D.Friedman [9]
} 
- First let us assign 1 to 1 then both c and d have to be 1 to justify l. However, these assignments would imply an $r=0$. Hence this leads to inconsistency.

- Now we backtrace to our previous decision point ie., $l=1$ and now take the other alternative of $k=1$. This justification will lead to a consistent solution. The input pattern is given by $111 \mathrm{X} 110$ or $11 \mathrm{X} 1110$.

Hence as seen from the above example, whenever there are several alternatives to justify or propagate an error, one of them is selected. However, if the selection leads to contradicting values on any path we trace back and change the last decision. When ever we back trace we erase all the values resulting from the wrong decision.

There are several fault oriented Test Generation algorithms [10] like the D-algorithm, 9-V algorithm, PODEM etc. In our experiments we used the PODEM algorithm with the 9V logic [15] and here is a brief discussion about the PODEM algorithm.

\subsection{PODEM:}

It is a implicit enumeration algorithm used for the combinational circuits. In this approach all the primary input patterns are examined as tests for a given fault. Once a PI pattern that activates a fault is found the search process is terminated. If no PI pattern can be a test, the fault is considered untestable.

The enumeration process used in PODEM can be understood from the decision tree shown in figure 4.2 .

Initially all the PIs are at $\mathrm{X}$ value ie., they are unassigned. Then one of the PIs is assigned a value. Once a initial value is assigned to a node, it is marked as unflagged. The 


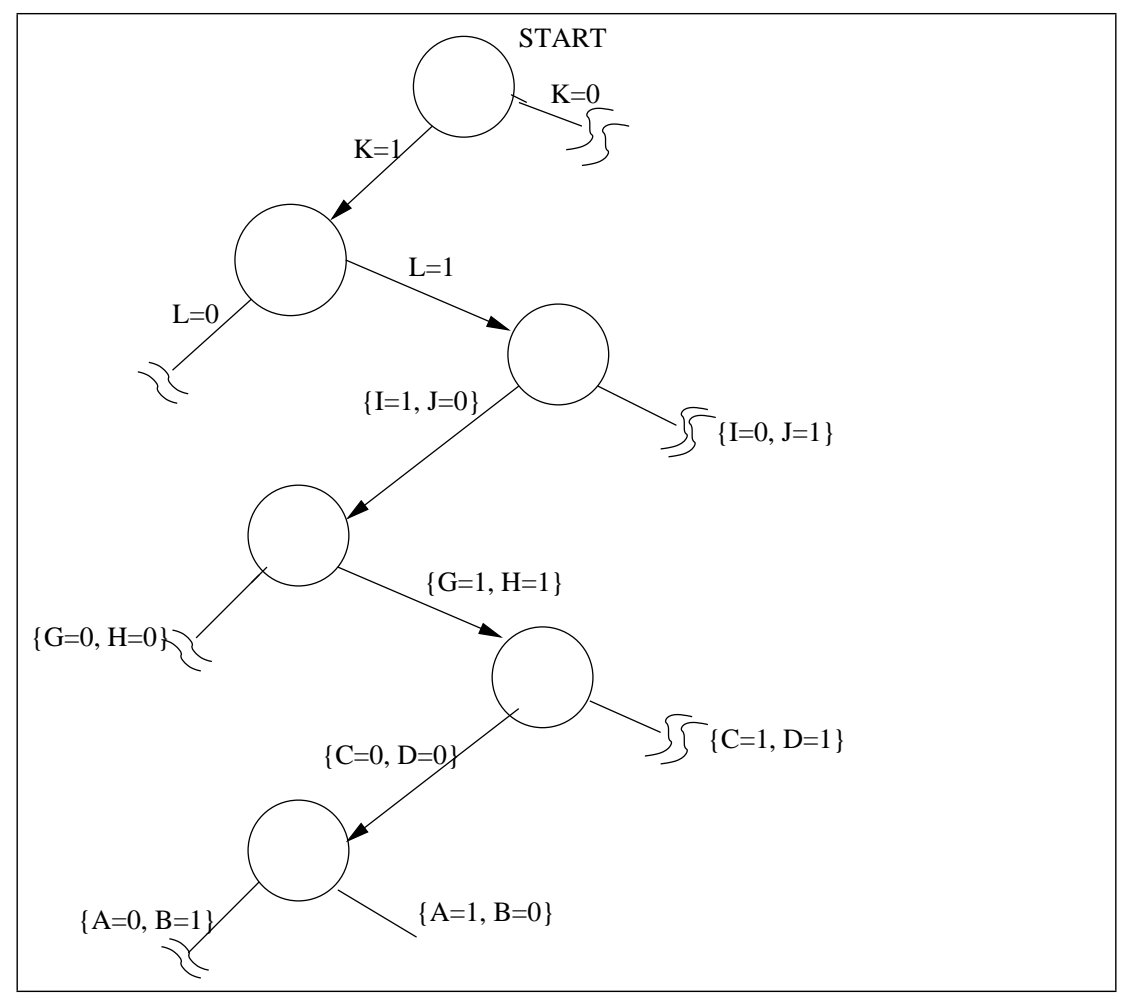

Figure 4.2: Decision Tree

new PI assignment is implied. The decision tree is a ordered list where the order of the nodes indicates the relative sequence in which the assignments were made and each node in the decision tree identifies an assignment of $0 / 1$ on a PI. If it is found from implication or assignment that the current assignment will not lead to a test, then the last decision is changed and the other value that was rejected earlier is taken now and the node is marked as flagged. When both the assignments are rejected then the current node is removed and the predecessor node's current assignment is also rejected. This process is repeated until a test is found. To determine the values on the PIs of a circuit first the objective is determined and then a backtrace is performed from the objective net to the Primary inputs. Several 
heuristics are used to perform the backtrace operation efficiently [30]. Initially the objective is to set the GUT to the fault value, once the GUT is set the objective is to propagate the fault closer to the primary output.

The high level algorithmic representation of the PODEM is shown in figure 4.3.

\subsection{ATPG for Sequential Circuits:}

The above discussion presents the details of ATPG for combinational circuits. In the case of sequential circuits the complexity increases as we need a sequence of vectors to detect a single stuck at fault.

In our experiments we used the ILA model for sequential circuits. A brief discussion of this model is presented bellow.

A ILA represents a sequential circuit in a combinational model. This is done by removing the FFs and repeating the combinational circuits between the FFs. This can be understood from the ILA model shown in figure4.4. Here each block represents the state of the circuit in one clock cycle and the number of blocks is equal to the sequence size. Each block has two types of inputs. One type of input is the PIs and the other are the present state lines $S_{i}$. Similarly there are two output vectors. One is the set of POs and the other is the set of next state lines $S(i+1)$.

Once the Sequential circuit is represented as a combinational model, the combinational ATPG can be used to generate the test patterns. ATPG activates the fault in the last time frame and justifies states in a reverse order. If the fault can be justified and propagated in the current time frame, continue with previous time frame. Repeat this procedure until 
we reach time frame 0

\subsection{Detection Scheme for Trojans:}

Trojan detection is done on the same lines as ssf detection. The main difference between a ssf and a Trojan as modeled here are

- Trojan has more than one input. Hence, justifying the Trojan inputs involves application of the PI patterns that result in a Trojan input vector that activates the Trojan For example consider the circuit C17 shown in figure 4.5. Here a Trojan (nand gate) is injected on node10. The Trojan has two inputs and hence we need to justify the values at these two inputs to activate the Trojan. The Primary input patterns that would detect the Trojan are $\{1=00,2=00,3=\mathrm{XX}, 6=00,7=\mathrm{XX}\},\{1=\mathrm{XX}, 2=00,3=00$, $6=\mathrm{XX}, 7=\mathrm{XX}\},\{1=11,2=\mathrm{XX}, 3=11,6=11,7=\mathrm{XX}\}$ and $\{1=11,3=11,6=00,2=11$, $7=\mathrm{XX}\}$ these patterns activate the Trojan. If the propagation of the Trojan output to the primary output is taken into consideration the input patterns that are needed are $\{1=00,2=00,3=\mathrm{XX}, 6=00,7=\mathrm{XX}\},\{1=\mathrm{XX}, 2=00,3=00,6=\mathrm{XX}, 7=\mathrm{XX}\},\{1=11$, $2=\mathrm{XX}, 3=11,6=11,7=\mathrm{XX}\}$

Now consider a sa0 fault at the same node in $\mathrm{C} 17$ circuit. The patterns that detect this fault are 00XXX or X00XX. It can be observed that the number of patterns for a Trojan are more than that of a ssf at the same node. The no of patterns increases with increasing number of Trojan inputs.

- Trojans can be sequential. For such Trojans the activation pattern is a sequence of Trojan input vectors. Hence we need to find the sequence of primary input vectors 
that would result in the required sequence of vectors at the Trojan inputs. A ssf can never be sequential.

\subsubsection{Modified PODEM Algorithm}

To accommodate the differences between a Trojan and ssf, the PODEM algorithm has to be modified. In the case of combinational Trojans the PODEM algorithm remains the same as that of a ssf. But we try to justify the Trojan inputs with the activation pattern and propagate the output of the Trojan to the Primary output.

Let the Trojan have $\mathrm{n}$ inputs. The activation pattern can be one or more vectors from the set of vectors $(000 \ldots . .000), \ldots . . .(111 \ldots 111)$. Initially we try to justify a Trojan with activation pattern $0000 \ldots 0$. If we are able to justify and propagate the Trojan o/p to the primary $\mathrm{o} / \mathrm{p}$ of the circuit the PI input pattern is added to the set of test patterns and the Trojan with activation pattern $0000 \ldots 0$ is said to be detectable. Then we proceed to justify the next set of Trojan inputs (ie., 000...001).

If the algorithm fails to find at least one input pattern that can justify and propagate the Trojan output, we mark the Trojan with the activation pattern 000...000 as undetectable. Then the next Trojan input value of $000 \ldots 001$ is justified. This process is continued until we find the test patterns for all the Trojan inputs.

As mentioned earlier we used the PODEM algorithm for test pattern generation. Bellow is an example with the generation procedure as mentioned above. The maximum distance of Trojan input from the Trojan output is considered to be two.

ExampleGeneration of test patterns for the Trojan injected on node 12

A Trojan is modeled on node 12 as shown in figure 4.6. As node 12 is a fanout, 
node 11 is used to measure the distance of various nodes of the circuit and hence to find the Trojan inputs. Here the distance of the Trojan inputs from the Trojan gate is considered to be 2 . Hence the possible set of Trojan inputs are $\{10,7,2\}$. Nodes that are at a level greater than node 12 are not considered as they cause a feedback. To generate test patterns we need to justify the Trojan inputs. As discussed above, we first try to generate test patterns for the case where the activation pattern is 000. The steps involved in the justification are as discussed bellow (9 valued logic is used in this example).

Initially we try to justify a 00 on node 10 . We trace back from node 10 to find the PI input pattern that would result in a 00 on node 10 . Heuristics are used for the selection of nodes during backtrace. Many heuristics have been proposed till date. Initially node 1 is assigned a 11 and an implication is performed. Next node 3 is assigned a 11 and implied. After this assignment and implication the states of various nodes are as shown in 4.6

Next a 00 is justified on node 7 and implication is performed. The resulting states of various nodes are as shown in the figure 4.7. Finally a 00 is justified and implied on node 2. The logic values at various nodes are as shown in figure 4.8 .

A 000 cannot be justified as there is no $\mathrm{X}$ path to propagate the Trojan output to the primary output. Hence it is marked as redundant. It can be observed that 001 is also redundant. Therefore we proceed to justify 010. Bellow are the various steps involved in the justification process.

A 00 on node 10 can be justified as discussed above, the states of various nodes after this justification are shown in figure4.6. Then we try to justify a 11 on the next Trojan input which is node 7 . As node 7 is a Primary Input we assign 11 to it and imply. The state of the circuit after this assignment and implication is as shown in figure 4.9 
Next node 2 is assigned 00 and implication is performed. The state of the circuit after this is shown in figure 4.10. Now there is a xpath from the Trojan output to the primary output of the circuit. Hence this Trojan is detectable.

To detect sequential Trojans we need a sequence of patterns that activate the Trojan. The size of the Trojan activation sequence depends on the number of flip-flops inside the black boxed Trojan and on the behavior of the circuit. Determining the length of the Trojan input sequence is crucial for its detection. In our model here we assume a sequence of size k. The algorithm for the modified form of PODEM algorithm for Trojan detection is shown in the flowchart 4.11. It can be seen from the flowchart 4.11 that once a pattern is found to detect the Trojan it is added to the sequence of vectors. Then it is checked to see if the sequence size found is equal to $\mathrm{k}$. If it is not equal to $\mathrm{k}$ we try to find the next value in the sequence. Once the sequence size is equal to $\mathrm{k}$ we exit and add the sequence to the list of sequences that detect the Trojan. 4.11

Note:The circuit in which the Trojan is injected can be sequential. Hence there can be cases of a combinational Trojan in sequential circuit and sequential Trojan in combinational circuit. For sequential circuits we need to use the ILA model discussed in section [4.3]. However in practice the scan chain model is used by many companies for testing and sequential circuits occur rarely during testing Hence we do not discuss these cases here. But Trojan detection technique presented here can be used to detect Trojans in a sequential circuit using ILA model. 


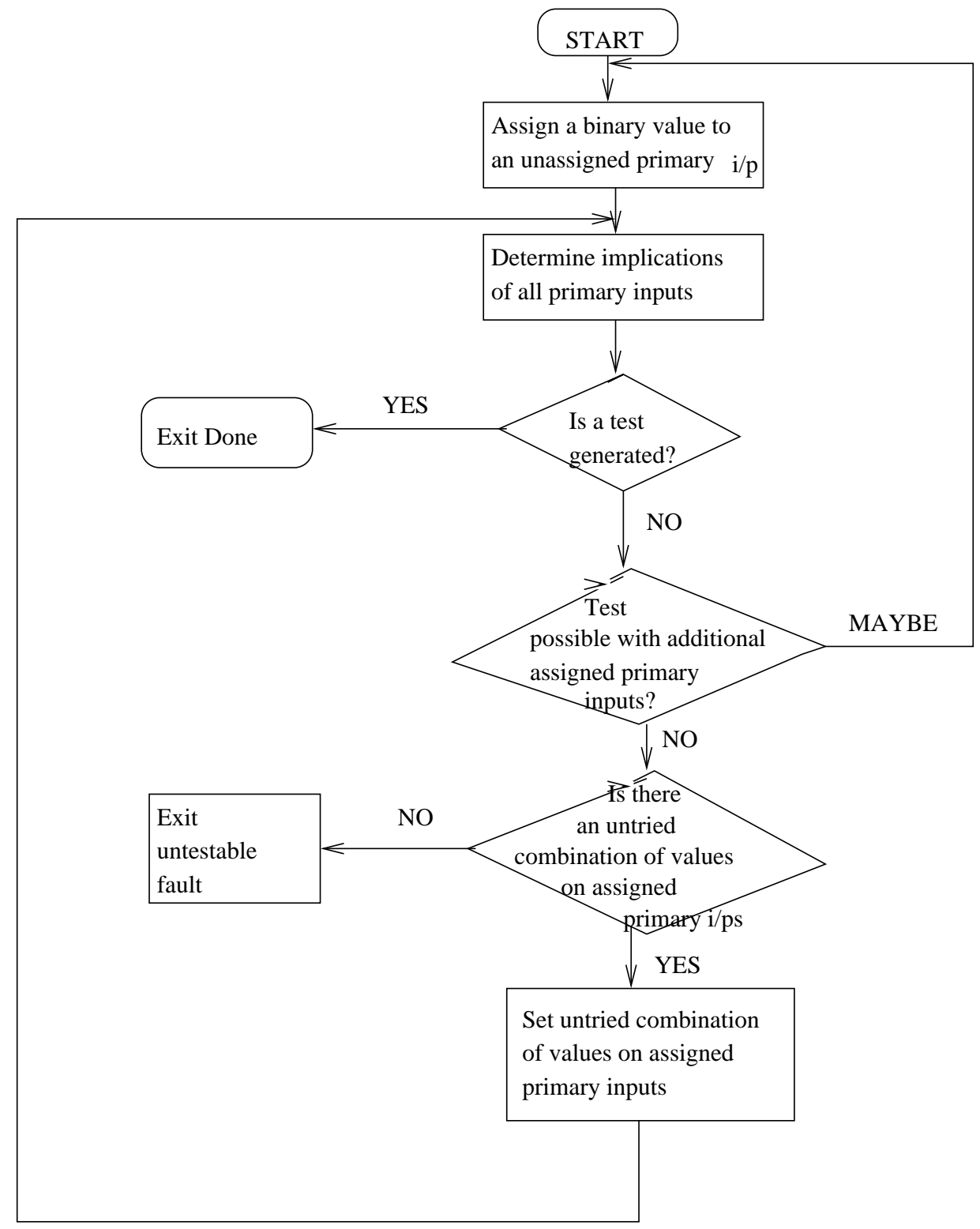

Figure 4.3: High Level Representation of PODEM Algorithm 


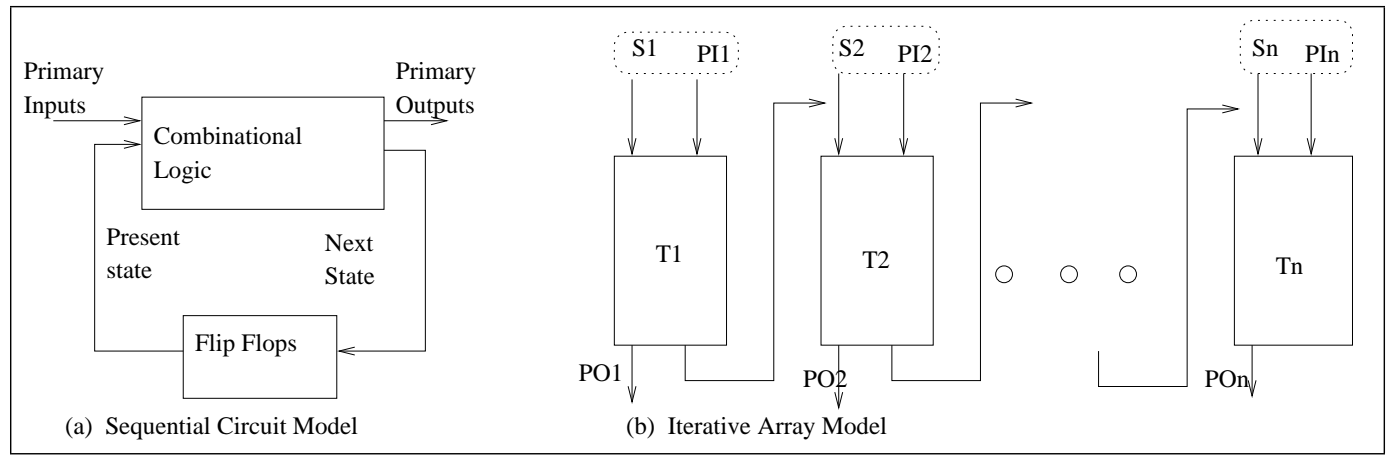

Figure 4.4: Iterative Logic Array

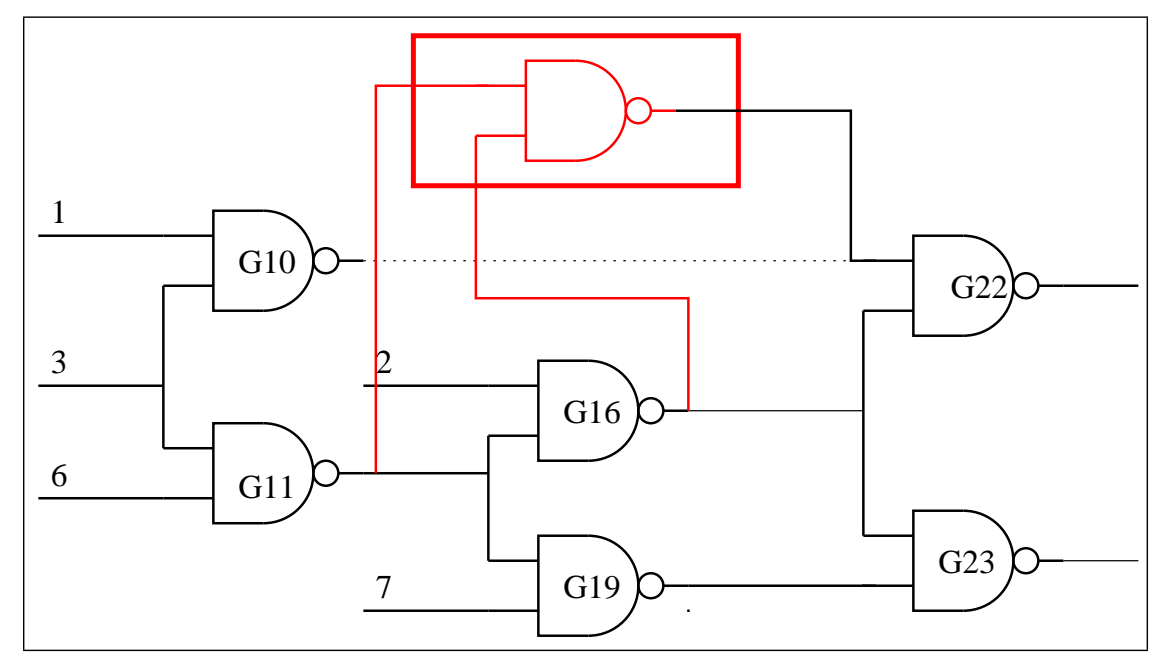

Figure 4.5: Circuit with Trojan Injected 


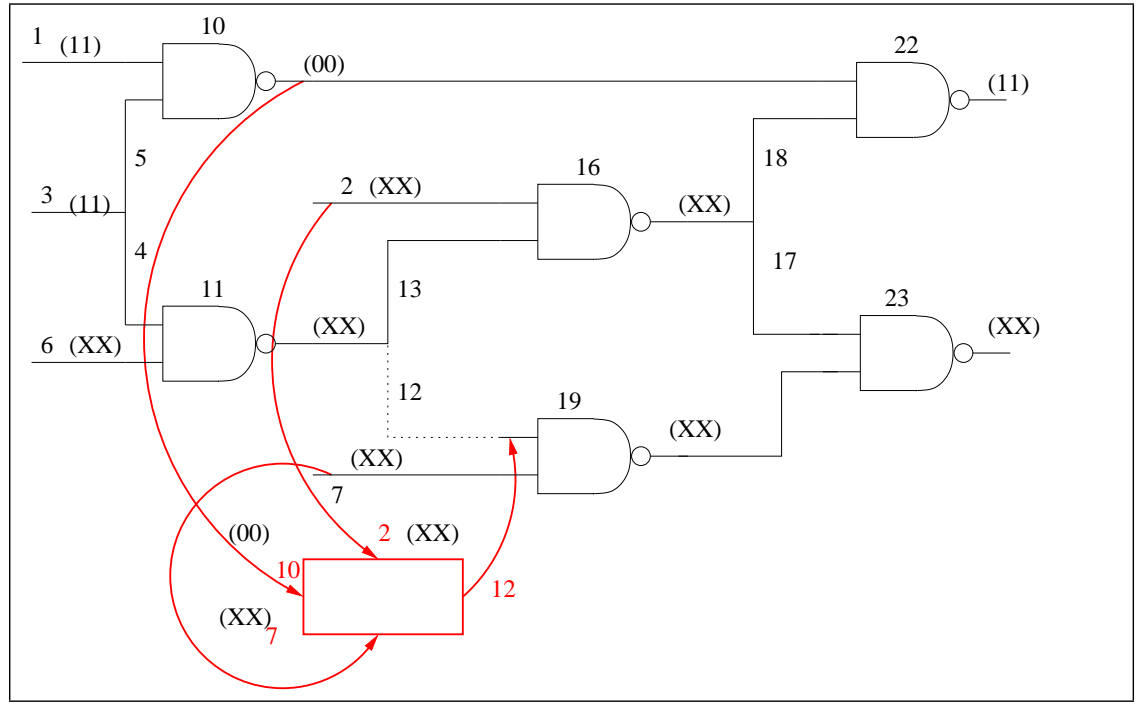

Figure 4.6: Test pattern generation for Trojan

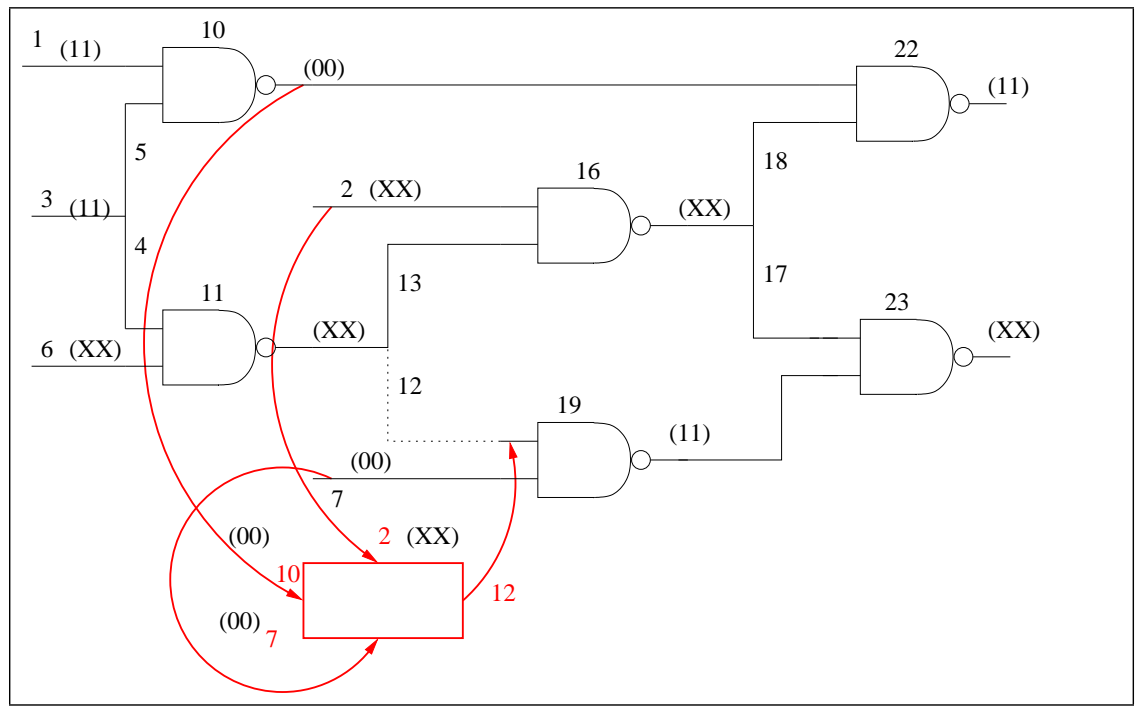

Figure 4.7: Test pattern generation for Trojan 


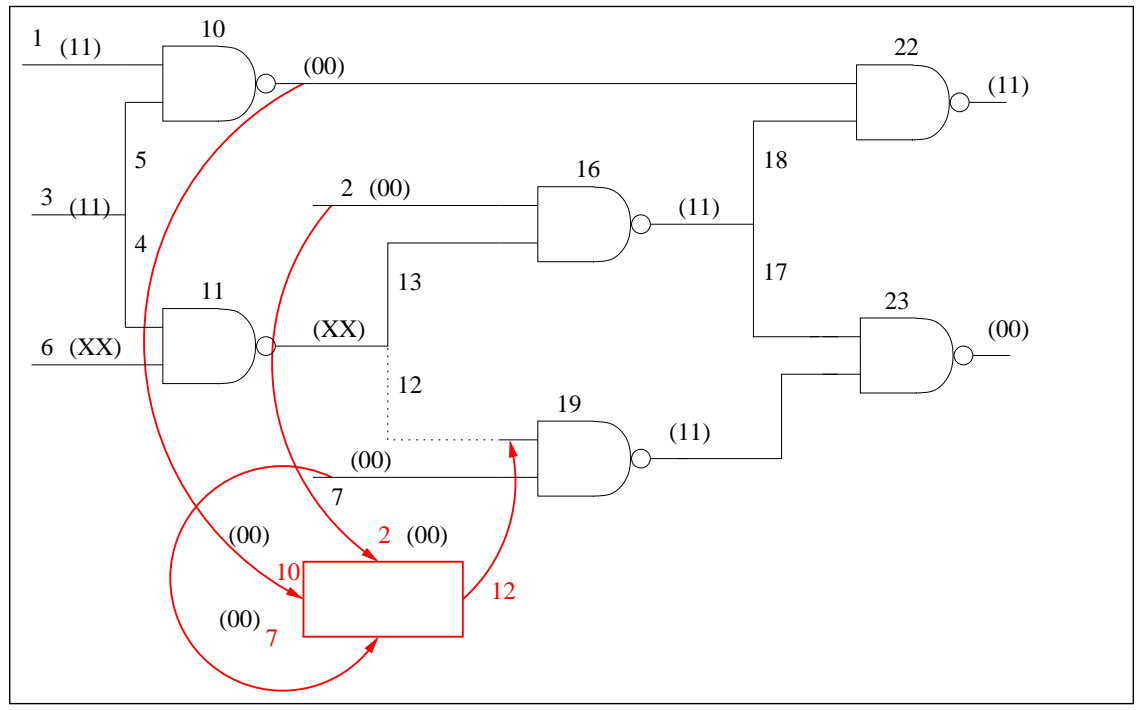

Figure 4.8: Test pattern generation for Trojan

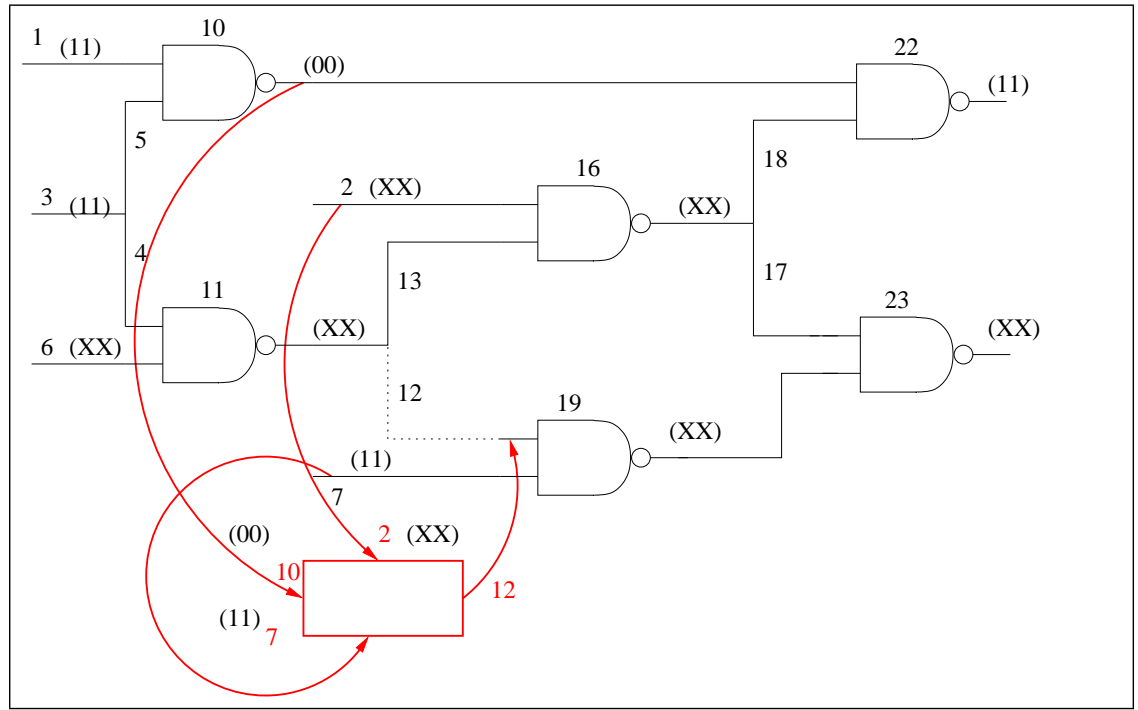

Figure 4.9: Test pattern generation for Trojan 


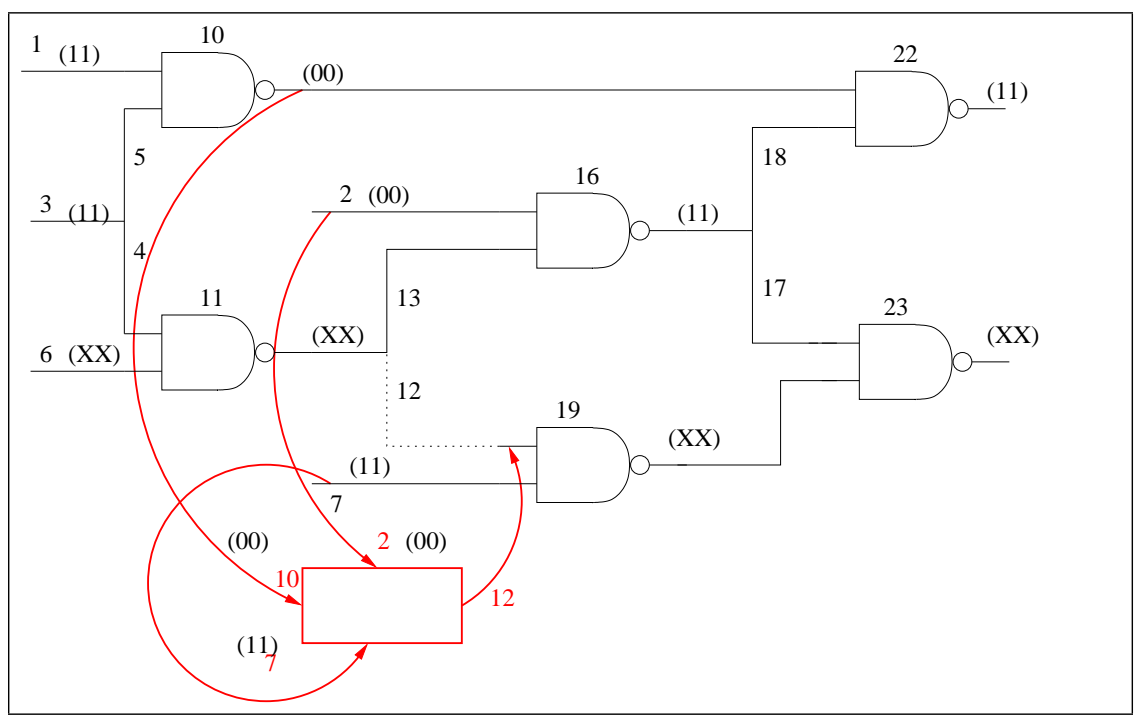

Figure 4.10: Test pattern generation for Trojan 


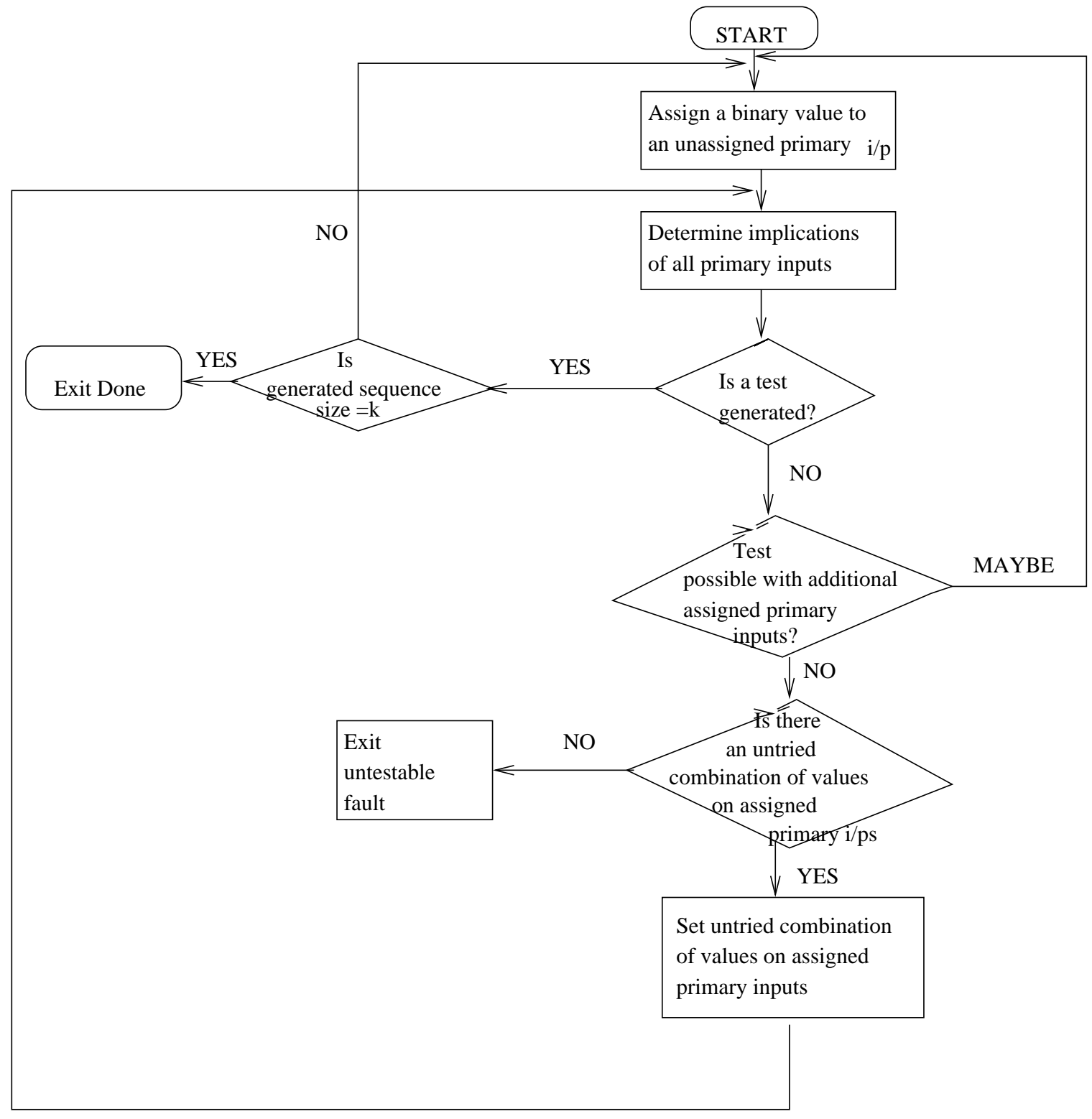

Figure 4.11: Modified PODEM Algorithm for the detection of Trojans 


\section{Chapter 5}

\section{Results}

A C program was developed to implement the modeling and detection techniques presented in the earlier chapters. The test patterns are generated for both combinational and sequential Trojans in various ISCAS'85 benchmark circuits [8] [20]. The complexity of the test generation process increases with the number of Trojan inputs. For example, consider a Trojan with $\mathrm{n}$ inputs, the size of the Trojan behavior is $9^{n}$. Here in our experiments we limited the number of inputs to 5 .

The efficiency of the generated test patterns in detecting Trojans is tested by injecting Trojans in some of the benchmark circuits and performing fault simulation. Here in the experiments care is taken to inject Trojans such that they are activated rarely. For this the frequency of occurrence of the activation pattern on the Trojan inputs and the observability of

the Trojan injected net are taken into account. The details of the Trojan injection procedure are presented in section 5.2.1. 


\subsection{Analysis of generated patterns}

The statistics of the test patterns generated are summarized in table 5.1. The column Detected indicates the number of patterns that would detect at least one Trojan when applied. The Redundant and Dropped columns indicate the number of redundant and dropped patterns. For example for the circuit c432 the number of detectable patterns generated are 3158 and the number of those redundant and dropped are 1320 and 4166. The number of boxed Trojans are 255, this means that modeled Trojans are injected in 255 locations and PODEM algorithm is used to generate the test patterns. The number of inputs varies from Trojan to Trojan and the average number of the inputs is mentioned as 4.43. Hence the average number of input patterns that can be applied to a Trojan are $2^{4.43}$.

Similar procedure is repeated when sequential Trojans are injected. The results are summarized in tables 5.3 and 5.2 for Trojans with sequence size two and three respectively. It can be observed that the number of patterns increases exponentially with the increase in the length of the Trojan input sequence. These results are represented in graphs (5.1 and 5.2 ) for a better comparison.

\subsection{Fault Simulation}

We first injected Trojans in some of the benchmark circuits using the procedure explained in section 5.2.1. Then fault simulation was performed using the generated test patterns to measure their performance. 
Table 5.1: Iscas85: Modeled Trojan

\begin{tabular}{|c|ccccc|}
\hline Circuit & boxed & avg. & Detected & Redundant & Droppede \\
\hline c432 & 255 & 4.43 & 3158 & 1320 & 4166 \\
c499 & 296 & 5.00 & 4316 & 0 & 7652 \\
c880 & 507 & 4.11 & 5084 & 2711 & 7223 \\
c1908 & 1420 & 3.81 & 8036 & 11230 & 16576 \\
c2670 & 1850 & 3.90 & 11842 & 15924 & 19576 \\
c3540 & 2633 & 4.08 & 15274 & 40011 & 23261 \\
c6288 & 4288 & 3.78 & 17042 & 9859 & 53207 \\
c7552 & 5836 & 4.04 & 32480 & 73654 & 57212 \\
s298 & 159 & 5.08 & 1460 & 5828 & 40 \\
s344 & 233 & 3.69 & 1171 & 3155 & 604 \\
s349 & 236 & 3.71 & 1175 & 3323 & 604 \\
s382 & 220 & 4.66 & 2477 & 5088 & 925 \\
s386 & 272 & 5.05 & 2057 & 9968 & 625 \\
s400 & 231 & 4.71 & 2618 & 5431 & 1073 \\
s444 & 262 & 4.51 & 2513 & 6030 & 877 \\
s641 & 484 & 3.07 & 3049 & 4788 & 2747 \\
s713 & 537 & 3.22 & 3167 & 5494 & 3561 \\
s820 & 475 & 5.55 & 4126 & 21259 & 697 \\
s832 & 481 & 5.56 & 4066 & 21578 & 780 \\
s1196 & 808 & 4.96 & 6887 & 25034 & 3583 \\
s1423 & 909 & 3.49 & 5317 & 5726 & 6453 \\
s5378 & 3921 & 3.25 & 19905 & 43874 & 9335 \\
s9234 & 7327 & 2.88 & 27141 & 71130 & 23393 \\
s15850 & 11971 & 2.71 & 47957 & 83668 & 36579 \\
s35932 & 21249 & 3.23 & 107940 & 119847 & 94911 \\
\hline
\end{tabular}


Table 5.2: Iscas89: Modeled Trojan k=3

\begin{tabular}{|c|ccccc|}
\hline Circuit & boxed & avg. & Detected & Red $^{1}$ & Drop \\
\hline c1355 & 856 & 3.56 & 1331168 & 30848 & 334036 \\
c1908 & 1420 & 3.56 & 2285555 & 336460 & 363495 \\
c2670 & 1850 & 3.65 & 3940509 & 390899 & 577349 \\
c3540 & 2633 & 3.74 & 3748156 & 1500411 & 679200 \\
c5315 & 3878 & 4.03 & 9949513 & 2604847 & 434218 \\
c6288 & 4288 & 3.67 & 906651 & 304808 & 1254254 \\
c7552 & 5836 & 3.75 & 6557851 & 2848756 & 1206041 \\
s344 & 233 & 3.53 & 171395 & 96759 & 10684 \\
s420 & 269 & 4.11 & 338301 & 164118 & 12541 \\
s510 & 333 & 4.51 & 651686 & 431700 & 3622 \\
s526 & 305 & 4.67 & 1338914 & 373951 & 9079 \\
s713 & 537 & 2.93 & 899056 & 95113 & 33184 \\
s838 & 561 & 4.05 & 715293 & 270509 & 42781 \\
s953 & 628 & 4.43 & 1119760 & 637091 & 29346 \\
s1196 & 808 & 4.39 & 1809050 & 909036 & 135504 \\
s1423 & 909 & 3.35 & 977312 & 261334 & 195334 \\
s1488 & 1106 & 4.66 & 2772186 & 1494555 & 35934 \\
s1494 & 1110 & 4.69 & 2806099 & 1477220 & 20094 \\
s5378 & 3921 & 3.09 & 3931671 & 1328027 & 371754 \\
s9234 & 7327 & 2.73 & 4216337 & 1615150 & 404607 \\
s13207 & 9966 & 2.60 & 7392631 & 2417688 & 119823 \\
s35932 & 21249 & 3.13 & 25906082 & 4221865 & 25906082 \\
\hline
\end{tabular}


Table 5.3: Iscas85: Modeled Trojan $\mathrm{k}=2$

\begin{tabular}{|c|ccccc|}
\hline Ckt no & num trojans & avg support & redundant & dropped & detected \\
\hline c1355 & 856 & 3.766355 & 12320 & 60592 & 124592 \\
c1908 & 1420 & 3.814789 & 53728 & 79623 & 237795 \\
c2670 & 1850 & 3.901622 & 96136 & 106035 & 366393 \\
c3540 & 2633 & 4.085074 & 331721 & 133764 & 300245 \\
c499 & 296 & 5.000000 & 0 & 25668 & 156576 \\
c5315 & 3878 & 4.449459 & 533406 & 173014 & 944836 \\
c7552 & 5836 & 4.042838 & 529004 & 319813 & 695011 \\
s1196 & 808 & 4.967822 & 191814 & 27636 & 163557 \\
s1238 & 819 & 5.354090 & 215349 & 27329 & 178818 \\
s13207 & 9966 & 2.755368 & 537950 & 38750 & 802520 \\
s1423 & 909 & 3.495049 & 39266 & 33528 & 88251 \\
s1494 & 1110 & 5.445045 & 330349 & 5346 & 253840 \\
s15850 & 1110 & 5.445045 & 330349 & 5346 & 253840 \\
s344 & 233 & 3.690987 & 18517 & 1906 & 17032 \\
s349 & 236 & 3.716102 & 19222 & 1906 & 17011 \\
s35932 & 236 & 3.716102 & 19222 & 1906 & 17011 \\
s386 & 272 & 5.051471 & 66975 & 2163 & 55635 \\
s444 & 262 & 4.515267 & 43679 & 2809 & 92187 \\
s510 & 333 & 5.096096 & 98231 & 579 & 58638 \\
s526 & 305 & 5.426229 & 109819 & 1361 & 93779 \\
s5378 & 3921 & 3.256312 & 293607 & 59035 & 382013 \\
s713 & 537 & 3.225326 & 27707 & 11345 & 99543 \\
s838 & 561 & 4.397504 & 59652 & 12462 & 61629 \\
s9234 & 7327 & 2.883035 & 384184 & 75386 & 418109 \\
s953 & 628 & 4.869427 & 134288 & 5632 & 86629 \\
\hline
\end{tabular}




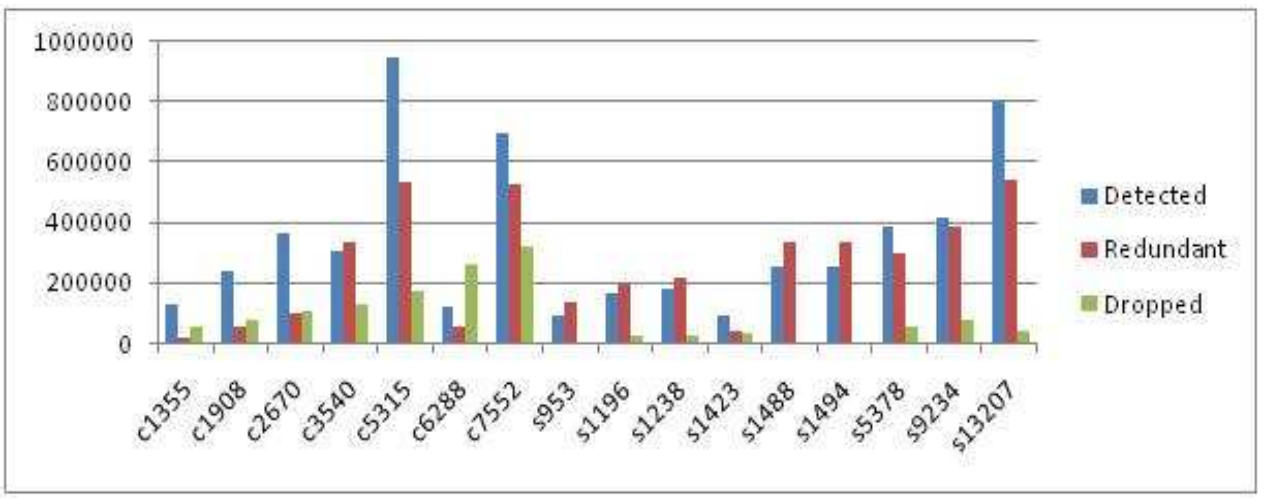

Figure 5.1: Results for sequential Trojans with $\mathrm{k}=2$

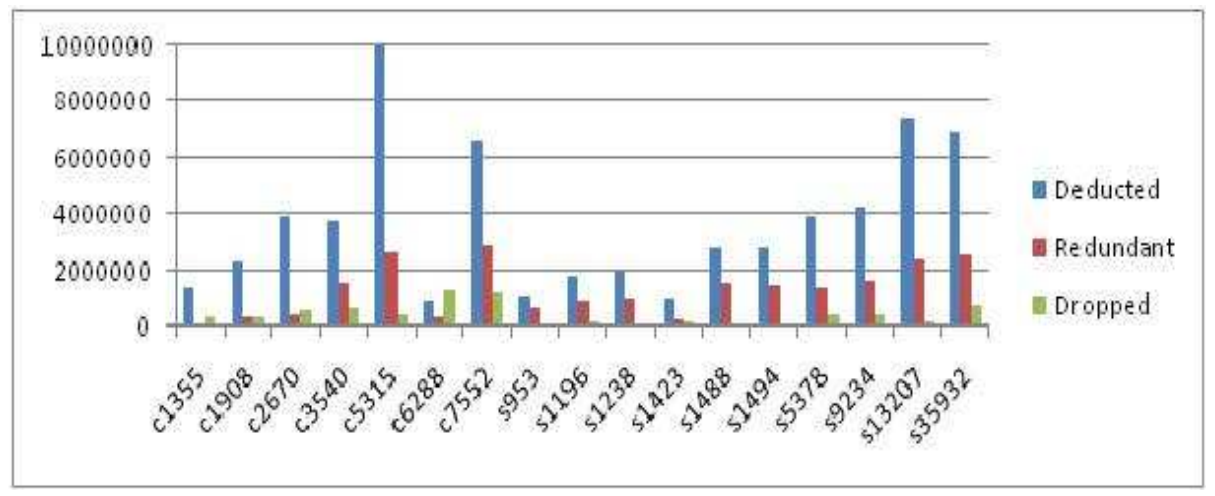

Figure 5.2: Results for sequential Trojans with $\mathrm{k}=3$

\subsubsection{Trojan Insertion Model}

Trojans are small modifications to circuits and are injected on the internal nodes of a circuit such that they are activated rarely. Hence the circuit functions normally for all the input patterns other than the activation pattern. The frequency of occurrence of the activation pattern is usually very low.

In our experiments to inject a Trojan we followed a two step procedure. 
step 1: Determine the inputs of a Trojan based on the frequency of occurrence of the activation pattern.

step 2: Select the potential nets to inject Trojans based on their observability.

Bellow is a detailed description of the procedure for the selection of the Trojan inputs and the potential nets for Trojan insertion.

\section{Trojan input selection}

Consider a Trojan circuit with d inputs. To inject this Trojan we first simulate the circuit with randomly generated input patterns and find the frequency of each input combination on d-tuple nets separately. The d-tuple nets with the lowest frequency input combination are chosen as the Trojan inputs.

Let $v_{1} \ldots v_{d}$ be an input combination, $g_{1}, \ldots, g_{d}$ be a $d$-tuple net set, $f_{g_{1}, g_{2}, \ldots, g_{d}}^{v_{1} v_{2} \ldots v_{d}}$ be the frequency of observing $g_{1}=v_{1} \wedge g_{2}=v_{2} \wedge \ldots \wedge g_{d}=v_{d}$ where $g_{i}$ is the $i^{\text {th }}$ net in the target circuit and $v_{i}$ is its logic value, and $T\left(g_{1}=v_{1}, \ldots, g_{d}=v_{d}\right)$ be the set of patterns that sets $g=v_{1} \wedge \ldots \wedge g_{d}=v_{d}$. Also, let $F(s)=\left\{\left\{g_{i}=v_{1}, g_{j}=v_{2}, \ldots, g_{k}=v_{d}\right\} \mid f_{g_{i}, g_{j}, \ldots, g_{k}}^{v_{1} v_{2} \ldots v_{d}}=s\right\}$. We explain this notation with an example below.

Example Exhaustive simulation c17 in Fig. 5.3 results in the following lowest frequencies for $d=2: f_{10,16}^{00}=2, f_{10,19}^{00}=2, f_{2,22}^{10}=2$ and $f_{10,23}^{01}=3$. Thus, $F(2)=$ $\{\{G 10=0, G 16=0\},\{G 10=0, G 19=0\},\{G 2=1, G 22=0\}\}$ and $F(3)=\{\{G 10=0, G 23=1\}\}$.

Also, $T(G 10=0, G 16=0)=\{1110 x\}, T(G 10=0, G 19=0)=\{1 x 101\}, T(G 2=1, G 22=0)=$ $\{0111 x\}$, and $T(G 10=0, G 23=1)=\{1110 x, 1 x 101\}$. Based on these results, we pick one element from $F(2)$ as the set of activation signals for the Trojan circuit. 


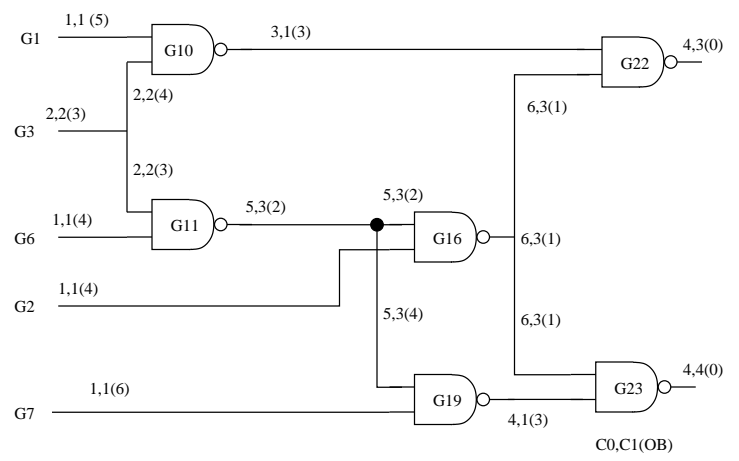

Figure 5.3: Observability and controllability values

In a circuit with $N$ nets the number of d-tuple nets is given by $\left(\begin{array}{c}N \\ d\end{array}\right)$ and the number of combinations on the d-tuple nets is $2^{d}$. For large values of $d$ it is impractical to calculate the frequencies of all combinations on d-tuple nets. This problem can be avoided by first simulating the circuit with $d=1$ and collecting the frequency of all the nets. Then identify $K$ nets with lowest frequency and resimulate the circuit to collect frequencies of activation signal sets that involve only the selected $\mathrm{K}$ nets.

\section{Trojan Signal Selection}

A net with low observability indicates that it is difficult to propagate the value at the gate to circuit output. Hence the line $\mathrm{L}$ with high observability value is identified as a potential net for Trojan insertion. Let $T(L=0)\{T(L=1)\}$ be the set of patterns that set the line $\mathrm{L}$ to $0\{1\}$ and let $\mathrm{T}(\mathrm{e})$ be the pattern that activates the Trojan. A net $\mathrm{L}$ is selected for Trojan insertion only if there is atleast one pattern that would activate the Trojan and also cause an inversion on the line L. In case, the selection of activation signal set $e$ does not satisfy 
the condition for the desired effect, we can either pick the next lowest frequency signal set or change the Trojan signal to a net with the next largest observability value.

Example We compute 0 - and 1-controllability $(C 0, C 1)$ and observability $(O B)$ values for c17 in Fig. 5.3. We compute these testability measures using Goldstein's method [10] with two minor modifications: (1) We add the number of fanouts at a stem to both of its controllability values if the number of fanouts is at least two, and (2) Cost of a gate is assumed to be zero in the calculation of controllabilities and observabilities. With this method, we found that G7 has the highest observability value, and $T(G 7=1)=\{x x x x 1\}$ and $T(G 7=0)=\{x x x x 0\}$. For activation signal set $e=\{G 10=0, G 16=0\}, T(e) \cap T(G 7=$ $1)=\{1110 x\} \cap\{x x x x 1\}=\{11101\}, T(e) \cap T(G 7=0)=\{1110 x\} \cap\{x x x x 0\}=\{11100\}$. However, for $L=G 1 e=\{G 2=1, G 22=0\}, T(e) \cap T(G 1=1)=\{0111 x\} \cap\{1 x x x x\}=\phi$. Therefore, this line cannot be selected since the corresponding condition does not hold.

\subsubsection{Results of Fault Simulation}

In our experiments we injected 16 Trojans in some of the benchmark circuits. The table 5.4 indicates the fault simulation results obtained for combinational Trojans. For example, if we consider the circuit c432 in table 5.4, out of the 16 injected Trojans all the 16 of them are

detected. Similar fault simulation is carried out for sequential Trojans with sequence size of 3 and the results are summarized in table 5.5. 
Table 5.4: Iscas85: Trojan Detection

\begin{tabular}{|c|ccc|}
\hline Circuit & Injected & Detected & Redundant \\
\hline $\mathrm{c} 432$ & 16 & 16 & 0 \\
$\mathrm{c} 499$ & 16 & 16 & 0 \\
$\mathrm{c} 880$ & 16 & 16 & 0 \\
$\mathrm{c} 1908$ & 16 & 12 & 3 \\
$\mathrm{c} 1355$ & 16 & 16 & 0 \\
$\mathrm{c} 2670$ & 16 & 14 & 2 \\
$\mathrm{c} 3540$ & 16 & 13 & 3 \\
$\mathrm{c} 5315$ & 16 & 15 & 1 \\
$\mathrm{c} 6288$ & 16 & 16 & 0 \\
$\mathrm{c} 7552$ & 16 & 15 & 1 \\
$\mathrm{~s} 298$ & 16 & 15 & 1 \\
$\mathrm{~s} 344$ & 16 & 10 & 6 \\
$\mathrm{~s} 349$ & 16 & 11 & 5 \\
$\mathrm{~s} 382$ & 16 & 12 & 4 \\
$\mathrm{~s} 386$ & 16 & 7 & 7 \\
$\mathrm{~s} 400$ & 16 & 11 & 4 \\
$\mathrm{~s} 444$ & 16 & 5 & 9 \\
$\mathrm{~s} 641$ & 16 & 14 & 1 \\
$\mathrm{~s} 713$ & 16 & 14 & 2 \\
$\mathrm{~s} 832$ & 16 & 8 & 8 \\
$\mathrm{~s} 1196$ & 16 & 10 & 6 \\
$\mathrm{~s} 1423$ & 16 & 15 & 1 \\
$\mathrm{~s} 5378$ & 16 & 16 & 0 \\
$\mathrm{~s} 9234$ & 16 & 15 & 1 \\
$\mathrm{~s} 15850$ & 16 & 15 & 1 \\
$\mathrm{~s} 35932$ & 16 & 16 & 0 \\
$\mathrm{~s} 38417$ & 16 & 12 & 4 \\
\hline
\end{tabular}

Table 5.5: Iscas89: Trojan Detection

\begin{tabular}{|c|ccc|}
\hline Circuit & Injected & Detected & Redundant \\
\hline s5378 & 16 & 15 & 1 \\
s9234 & 16 & 14 & 2 \\
s13207 & 16 & 14 & 2 \\
s35932 & 16 & 16 & 0 \\
\hline
\end{tabular}




\section{Chapter 6}

\section{Conclusion}

This thesis has proposed a technique for detection of Trojans using a modified form of ATPG. The modelling of Trojans is done based on the proximity estimation, where nodes at a distance $=d_{\max }$ from the Trojan node are considered i to be the inputs to the Trojan. Then the Trojan inputs are justified with the activation pattern and the Trojan output is propagated to the primary output of the circuit. If a primary input pattern that could justify the Trojan inputs and propagate Trojan output to the primary output of the circuit is found then the Trojan with the given activation pattern is considered to be detected. In this report we tabulated the results of the test pattern generation and fault simulation for combinational or scan chained sequential circuits. It can be observed from the results that the test size increases almost exponentially with increase in the sequence size of a Trojan. The advantage

of this technique is that it can be implemented using the existing ATPG tools, it fits into the normal design flow and does not need any modifications to the IC design process. 


\section{Bibliography}

[1] Mainak Banga and Michael S. Hsiao. A region based approach for the detection of hardware trojans. IEEE International workshop on Hardware-Oriented Security and Trust, Pages 40-47, 2008

[2] Mainak Banga, Maheshwar Chandrasekhar, Lei Fang and Michael S.Hsiao. Guided test generation for isolation and detection of embedded trojans in ICs. Proceedings of the $18^{\text {th }}$ ACM Great Lakes Symposium on VLSI, pages 363-366, 2008

[3] Rajat S.Chakraborty, Francis Wolf, Christos Papachristou, Swarup Bhunia. Towards trojan-free trusted ics: Problem analysis and detection scheme. Proceedings of Design, automation and test conference 2008, Pages 1362-1365

[4] Dakshi Agarwal, Selcuk Baktir, Deniz Karakoyunlu, Pankaj Rohatgi and Berk Sunar. Trojan detection using IC fingerprinting. in Proc. IEEE Symp. on Security and Privacy,May 20-23, $200 \%$.

[5] G. Edward Suh and Srinivas Devadas. Physical unclonable functions for device authentication and secret key generation. Proceedings of the Design Automation Conference (DAC), pages 914, $200 \%$.

[6] Xiaoxiao Wang Tehranipoor and M Plusquellic. Detecting malicious inclusions in secure hardware: Challenges and solutions. IEEE International Workshop on Hardware-Oriented Security and Trust, Pages 15-19, 2008

[7] Xiaoxiao Wang, Hassan Salmani, Mohammad Tehranipoor and Jim Plusquellic. Hardware trojan detection and isolation using current integration and localized current analysis. IEEE Symp, On Defect and Fault Tolerance of VLSI Systems, pages 87-95, 2008

[8] F. Brglez and H. Fujiwara, "A Neutral Netlist of 10 Combinational Benchmark Circuits," Proc. IEEE ISCAS, 1985, pp. 695-698.

[9] Miron Abramovici, Melvin A.Breuer, Arthur D. Friedman Digital Systems Testing and Testable Design Jaico Publishing House, ISBN 8172248911 2006

[10] N.K.Jha and S.Gupta, Testing of Digital Systems Cambridge University Press, UK, 2003

[11] Prabhakar Goel An Implicit Enumeration Algorithm to Generate Tests for Combinational Logic Circuits Proc of Fault-Tolerant Computing, 1995, 27-30 Jun 1995 Pages 337-343

[12] W.T.Cheng, T.J. Chakraborty Gentest: An Automatic test generation system for sequential circuits. IEEE trans on computers, 22(4):43-49, Apr. 1989

[13] Trust in Integrated Circuit (TIC), http://www.darpa.mil/MTO/solicitations/baa07-24/index.html. February 07. 
[14] D. Hely, et al. Scan Design and Secure Chip, Proc. of the 10th IEEE Intl. On-Line Testing Symposium, 2004

[15] Charles W.Cha, william E. Donath, Fusun Ozgunner 9-V Algorithm for Test Pattern Generation of Combinational Digital Circuits IEEE Transactions on Computers archive, Pages 193-200, 1978

[16] Wu-Tung Cheng Current Status and Future Trend on CAD Tools for VLSI Testing Proceedings of the 9th Asian Test Symposium Page: 10, 2000

[17] Wu-Tung Cheng Split Circuit Model For Test Generation Proceedings of the 25th ACM/IEEE Design Automation Conference Pages: 96 - 101, 1988

[18] Defense Science Board Task Force. High performance microchip supply, http://www.acq.osd.mil/dsb/reports/2005-02-HPMSReportFinal.pdf. February 2005

[19] J.P. Roth, Diagnosis of automata failures: A calculus and a method. IBM J. Res. Develop., 10:278-291, July 1966.

[20] F. Brglez, D. Bryan, and K. Kozminski. "Combinational Profiles of Sequential Benchmark Circuits," IEEE ISCAS, May 1989.

[21] P. Kocher, R. Lee, G. McGraw, A. Raghunathan, and S. Ravi, Security as a New Dimension in Embedded System Design, in DAC, June 2004, pp. 753-760.

[22] J. Lee, M. Tehranipoor, C. Patelm and J. Plusquellic, "Securing Scan Design Using Lock and Key Technique," in Proc. of IEEE Symp. on DFT, 2005.

[23] J. Lee, M. Teharanipoor, and J. Plusquellic, "A Low-Cost Solution for Protecting IPs Against ScanBased Side-Channel Attacks" VTS 2006.

[24] D. Boneh, R. DeMillo, and R. Lipton, "On the importance of checking cryptographic protocols for faults," In EUROCRYPT '97, vol. 1233 of Lecture Notes in CS, pp.37-51. Springer-Verlag, 1997.

[25] K. Hafner, et al., "Design and Test of an Integrated Cryptochip," IEEE DET T, pp. 617, Dec. 1991.

[26] S. Mangard, M. Aigner, and S.Dominikus, "A highly regular and scalable AES hardware architecture," IEEE Trans. Comput., vol. 52, no. 1, pp. 483-491, Apr. 2003.

[27] I. Verbauwhede, P. Schaumont and K. Kuo, "Design and performance testing of a $2.29 \mathrm{~Gb} / \mathrm{s}$ Rijndael processor," in IEEE Journal of Solid-State Circuits, Mar. 2003, pp. 569-572.

[28] K. Tiri and I. Verbauwhede, "A VLSI Design Flow for Secure Side-Channel Attack Resistant ICs," in Proc. of DATE, Mar. 2005, pp. 58-63.

[29] S. Ravi, A. Raghunathan, and S. Chakradhar, "Tamper Resistance Mechanisms for Secure Embedded Systems," in Proc. of the 17th Intl. Conf. on VLSI Design, 2004, pp. 605-611.

[30] L. H. Goldstein and E. L. Thigpen, "SCOAP: Sandia Controllability/ Observability Analysis Program," in Proc. of the 17th Design Automation Conf., June 1980, pages 190-196

[31] B. Yang, K. Wu, R. Karri. Secure Scan: A Design-for-Test Architecture for Crypto Chips, IEEE Trans. on CAD, 2006, pp. 2287-2293.

[32] B. Yang, K. Wu, and R. Karri, "Scan Based Side Channel Attack on Dedicated Hardware Implementations of Data Encryption Standard," in Proc. IEEE ITC, 2004, pp. 339-344. 
[33] Carol Marsh ," A Security Tagging Scheme for ASIC Designs and Intellectual Property Cores" http://www.us.design-reuse.com/articles/article15105.html.200\%.

[34] R. Usselmann. aes cipher ip core. In http://www.opencores.org, 2002.

[35] R. Karri, K. Wu, and P. Mishra, "Fault-Based Side-Channel Cryptanalysis Tolerant Architecture for Rijndael Symmetric Block Cipher," in IEEE Intl. Symp. on DFT, 2001, pp. 427-435. 